\title{
Consistency Improvement With a Feedback Recommendation in Personalized Linguistic Group Decision Making
}

\author{
Cong-Cong $\mathrm{Li}^{\left({ }^{(}\right)}$, Haiming Liang ${ }^{(\mathbb{1})}$, Yucheng Dong ${ }^{(\mathbb{1}}$, Senior Member, IEEE, Francisco Chiclana ${ }^{(}$, \\ and Enrique Herrera-Viedma ${ }^{\circledR}$, Fellow, IEEE
}

\begin{abstract}
Consistency is an important issue in linguistic decision making with various consistency measures and consistency improving methods available in the literature. However, existing linguistic consistency studies omit the fact that words mean different things for different people, that is, decision makers' personalized individual semantics (PISs) over their expressed linguistic preferences are ignored. Therefore, the aim of this article is to propose a novel consistency improving approach based on PISs in linguistic group decision making. The proposed approach combines the characteristics of personalized representation and integrates the PIS-based model in measuring and improving the consistency of linguistic preference relations. A detailed numerical and comparative analysis to support the feasibility of the proposed approach is provided.
\end{abstract}

Index Terms-Consistency, group decision making (GDM), linguistic preference relation, personalized individual semantics (PISs).

Manuscript received June 18, 2020; revised October 23, 2020; accepted May 26, 2021. This work was supported in part by the NSF of China under Grant 71901182, Grant 71601133, and Grant 71871149; in part by Sichuan University under Grant sksyl201705 and Grant YJ201906; in part by Southwest Jiaotong University under Grant YJSY-DSTD201918; in part by the China Postdoctoral Science Foundation under Grant 2020M673283 and Grant 2682021ZTPY073; and in part by the Spanish State Research Agency under Project PID2019-103880RB-I00/AEI/10.13039/501100011033. This article was recommended by Associate Editor F. J. Cabrerizo. (Corresponding author: Yucheng Dong.)

Cong-Cong $\mathrm{Li}$ is with the School of Economics and Management and the Key Laboratory of Service Science and Innovation of Sichuan Province, Southwest Jiaotong University, Chengdu 610031, China (e-mail: congcongli@swjtu.edu.cn).

Haiming Liang is with the Center for Network Big Data and DecisionMaking, Business School, Sichuan University, Chengdu 610065, China (e-mail: hmliang@scu.edu.cn).

Yucheng Dong is with the Center for Network Big Data and DecisionMaking, Business School, Sichuan University, Chengdu 610065, China, also with the School of Information Management and Statistics, Hubei University of Economics, Wuhan 430205, China, and also with the School of Management, Shenzhen Institute of Information Technology, Shenzhen 518172, China (e-mail: ycdong@scu.edu.cn).

Francisco Chiclana is with the Institute of Artificial Intelligence, De Montfort University, Leicester LE1 9BH, U.K., and also with the Andalusian Research Institute on Data Science and Computational Intelligence, University of Granada, 18071 Granada, Spain (e-mail: chiclana@dmu.ac.uk).

Enrique Herrera-Viedma is with the Andalusian Research Institute in Data Science and Computational Intelligence, Department of Computer Science and AI, University of Granada, 18071 Granada, Spain, and also with the Department of Electrical and Computer Engineering, Faculty of Engineering, King Abdulaziz University, Jeddah 21589, Saudi Arabia (e-mail: viedma@decsai.ugr.es).

Color versions of one or more figures in this article are available at https://doi.org/10.1109/TCYB.2021.3085760.

Digital Object Identifier 10.1109/TCYB.2021.3085760

\section{INTRODUCTION}

$\mathbf{P}$ REFERENCE relation is the most commonly used preference representation structure in group decision making (GDM). There are various types of preference relations: additive preference relation [24], [33]; multiplicative preference relation [3], [25], [30]; and linguistic preference relation [9], [11].

In real decision-making activities, it is common that decision makers provide their knowledge and preferences using words (linguistically) rather than numbers (numerically). Generally, the consistency of information is important in GDM problems because a lack of it may lead to the inconsistent results [6]-[8], [18], [38], [42]. Existing studies in the literature measure the consistency of linguistic preference relations mainly by computing the difference between the original linguistic preferences and their estimated consistent ones [1], [21]. If the consistency of a linguistic preference relation is unacceptable, then methods to improve the consistency degree are applied. Generally, two types of consistency improving approaches are often used in decision making with linguistic preference relations [21].

1) the iterative approach, which improves the consistency degree by helping decision makers to construct a new linguistic preference relation according to the consistent linguistic preference relation;

2) the optimization method, which deals with inconsistent linguistic preference relation by finding a suitable linguistic preference relation with acceptable consistency to preserve the original information as much as possible.

Dong et al. [6] proposed an iterative algorithm to improve the consistency degree of linguistic preference relations by constructing a new linguistic preference relation with acceptable consistency, and also suggested a nonlinear programming model to improve the consistency. Jin et al. [17] proposed two automatic iterative algorithms to help decision makers improve additive consistency level until it is acceptable. Wu et al. [40] proposed an integer optimization model for improving consistency by deriving the acceptably consistent linguistic preference relation. More research regarding the consistency improving methods can be found in the recent review [21].

It is a fact that words mean different things for different people [26], [27]. Mathematically, this has been addressed 
in linguistic GDM by using type-2 fuzzy sets [26] and the multigranular linguistic model [14], [28]. Although they are useful in processing the multiple meanings of words, they are unable to represent the specific meaning of words for each decision maker. Therefore, the personalized individual semantics (PISs) model was proposed in [19] to obtain the personalized numerical scales of linguistic terms for decision makers. Furthermore, Li et al. [20], [22], Zhang et al. [43], and Tang et al. [34], [35] studied the consistency-driven approaches to show the PISs in hesitant linguistic GDM, large-scale linguistic GDM, and distribution linguistic GDM, respectively. The application of the PIS model was studied in failure modes and effects analysis [44] and opinion dynamics [23].

The PISs among decision makers can influence the measurement of consistency for linguistic expressions. For example, let $S=\left\{s_{0}=\right.$ very poor, $s_{1}=$ poor, $s_{2}=$ medium, $s_{3}=$ good,$s_{4}=$ very good $\}$ be an established linguistic term set. A decision maker who assesses the preference of alternative $x_{i}$ over alternative $x_{j}$ with the $s_{3}$ value, the preference of the alternative $x_{j}$ over the alternative $x_{z}$ with the $s_{2}$ value, and the preference of the alternative $x_{i}$ over the alternative $x_{z}$ with the $s_{2}$ value, is actually providing, based on the additive transitivity [32], [33] and the 2-tuple linguistic computational model [10], is additive consistent linguistic preferences on the set of alternatives $\left\{x_{i}, x_{j}, x_{z}\right\}$. However, if the PISs of words are considered, then these linguistic preferences may not satisfy the additive consistency requirement for some decision makers.

Although the existing consistency improving approaches have been investigated intensively, the decision makers' PISs are not considered. Therefore, this article revisits the linguistic consistency improving methodologies from the PISs perspective. Specifically, we propose a consistency improving method with a feedback recommendation based on PISs in linguistic GDM, in which the feedback recommendation helps decision makers revise their preferences to improve the consistency. The main goal of the proposed consistency improving method is to construct a new linguistic preference relation that has acceptable consistency taking into account the decision makers' PISs. This proposal includes the following stages.

1) By constructing a consistency-driven optimization model, personalized numerical scales of linguistic terms are set for different decision makers to personalize individual semantics; this is followed by the development of a novel consistency index of linguistic preference relations based on the PISs.

2) A PIS-based consistency improving method is proposed. A theoretical analysis shows that: a) the method's adjusted linguistic preference relations are of acceptable consistency and b) the convergence of the consistency improving process.

3) A comparative study with the existing consistency improving methods based on experimental simulations is included. The obtained results show that the integration of the PIS model can help improve the consistency of linguistic preference relations more rapidly.
The remainder of this article is arranged as follows. Section II introduces the necessary preliminaries to develop the proposed PIS-based consistency improving method of linguistic preference relation in Section III. Section IV includes numerical examples to illustrate the PIS-based consistency improving process, while Section V is devoted to an experimental comparative study of the proposed approach performance with respect to the existing approaches in the literature. Finally, Section VI concludes this article with final remarks.

\section{Preliminaries}

This section introduces preliminary material necessary to build the proposed consistency improving process: the 2-tuple linguistic model and the numerical scale with PISs.

\section{A. 2-Tuple Linguistic Model}

The 2-tuple linguistic model, proposed by Herrera and Martínez [10], is widely used in computing with word frameworks.

Definition 1 [10]: Let $S=\left\{s_{0}, s_{1}, \ldots, s_{g}\right\}$ be a linguistic term set, and $\beta \in[0, g]$ be a value representing the result of a symbolic aggregation operation. The 2-tuple linguistic model comprises the transformation function between symbolic aggregation numerical values and 2-tuples

$$
\begin{aligned}
& \Delta:[0, g] \rightarrow \bar{S} \\
& \Delta(\beta)=\left(s_{i}, \alpha\right)
\end{aligned}
$$

where $i=\operatorname{round}(\beta)$ and $\alpha=\beta-i, \alpha \epsilon[-0.5,0.5)$.

The 2-tuple negation operator is defined as $\operatorname{Neg}\left(\left(s_{i}, \alpha\right)\right)=$ $\Delta\left(g-\left(\Delta^{-1}\left(s_{i}, \alpha\right)\right)\right)$, where $\Delta^{-1}\left(s_{i}, \alpha\right)=i+\alpha$ is the inverse function of $\Delta$.

Linguistic preference relations, as defined below, are widely used in decision making.

Definition 2 [12], [13]: Let $S=\left\{s_{0}, s_{1}, \ldots, s_{g}\right\}$ be a linguistic term set. A linguistic preference relation on a set of alternatives $X=\left\{x_{1}, x_{2}, \ldots, x_{n}\right\}$ is represented by a matrix $L=\left(l_{i j}\right)_{n \times n}$, whose element $l_{i j} \in S$ is the preference degree of alternative $x_{i}$ over $x_{j}$, subject to $l_{i j}=\operatorname{Neg}\left(l_{j i}\right)$ for $i, j=1,2, \ldots, n$.

The consistency of a linguistic preference relation based on the 2-tuple linguistic model is measured as follows.

Definition 3 [1]: A linguistic preference relation on a linguistic term set $S, L=\left(l_{i j}\right)_{n \times n}$, is consistent if

$$
\Delta^{-1}\left(l_{i j}\right)+\Delta^{-1}\left(l_{j k}\right)-\Delta^{-1}\left(l_{i z}\right)=\frac{g}{2} \forall i, j, z=1,2, \ldots, n .
$$

The consistency index of $L$ is defined as follows:

$$
\begin{aligned}
\mathrm{CI}(L)= & 1-\frac{2}{3 g n(n-1)(n-2)} \sum_{i, j, z=1}^{n} \\
& \times\left(\Delta^{-1}\left(l_{i j}\right)+\Delta^{-1}\left(l_{j z}\right)-\Delta^{-1}\left(l_{i z}\right)-\frac{g}{2}\right) .
\end{aligned}
$$

A larger value of $\mathrm{CI}(L) \in[0,1]$ indicates a better consistency of $L$. 


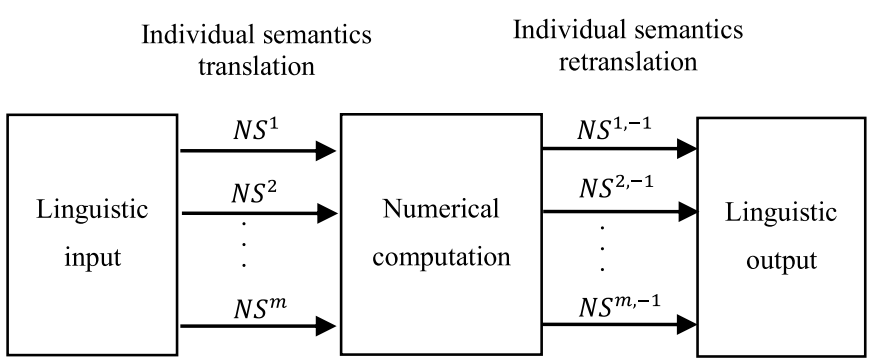

Fig. 1. Framework for the linguistic model with PISs.

\section{B. PIS Based on Numerical Scale}

Dong et al. [4] extended the 2-tuple linguistic model with the concept of the numerical scale.

Definition 4 [4]: Let $S=\left\{s_{0}, s_{1}, \ldots, s_{g}\right\}$ be a linguistic term set, and $\mathbb{R}$ be the set of real numbers. A function NS $: S \rightarrow \mathbb{R}$ is called a numerical scale of $S$, and $\operatorname{NS}\left(s_{i}\right)$ is referred to as the numerical index of $s_{i}$.

If $\mathrm{NS}\left(s_{i}\right)<\mathrm{NS}\left(s_{i+1}\right)(\forall i=0,1, \ldots, g-1)$, then the numerical scale NS on $S$ is ordered.

Note 1: The concept of the numerical scale was first proposed in [4]. The established range of the numerical scale will not influence its essence, and in the original definition [4], the value of the numerical scale is defined on the real number set in a general way, which provides a connect framework for computing with words [5]: setting $\mathrm{NS}\left(s_{i}\right)=i(i=0,1, \ldots, g)$ yields the 2-tuple linguistic model [10]; setting $\operatorname{NS}\left(s_{i}\right)=$ $C C V\left(s_{i}\right)(i=0,1, \ldots, g)$ yields the Wang and Hao model [36]; and setting $\mathrm{NS}\left(s_{i}\right)=\Delta^{-1}\left(s_{I^{\prime}(i)}^{n\left(t_{m}\right)}\right)(i=0,1, \ldots, g)$ yields the unbalanced linguistic model [15].

Definition 5 [4]: Let $S$ be defined as above. The 2-tuple numerical scale NS $: \bar{S} \rightarrow \mathbb{R}$ is

$$
\mathrm{NS}\left(s_{i}, \alpha\right)=\left\{\begin{array}{l}
\mathrm{NS}\left(s_{i}\right)+\alpha \times\left(\mathrm{NS}\left(s_{i+1}\right)-\mathrm{NS}\left(s_{i}\right)\right), \alpha \geq 0 \\
\mathrm{NS}\left(s_{i}\right)+\alpha \times\left(\mathrm{NS}\left(s_{i}\right)-\mathrm{NS}\left(s_{i-1}\right)\right), \alpha<0
\end{array}\right.
$$

The inverse of a 2-tuple numerical scale NS is $\mathrm{NS}^{-1}: \mathbb{R} \rightarrow \bar{S}$

$$
\mathrm{NS}^{-1}(r)=\left\{\begin{array}{c}
\left(s_{i}, \frac{r-\mathrm{NS}\left(s_{i}\right)}{\mathrm{NS}^{k}\left(s_{i+1}\right)-\mathrm{NS} S^{k}\left(s_{i}\right)}\right), \quad \mathrm{NS}\left(s_{i}\right)<r<\frac{\mathrm{NS}\left(s_{i}\right)+\mathrm{NS}\left(s_{i+1}\right)}{2} \\
\left(s_{i}, \frac{r-\mathrm{NS}\left(s_{i}\right)}{\mathrm{NS}\left(s_{i}\right)-\mathrm{NS}\left(s_{i-1}\right)}\right), \frac{\mathrm{NS}\left(s_{i-1}\right)+\mathrm{NS}\left(s_{i}\right)}{2} \leq r \leq \mathrm{NS}\left(s_{i}\right) .
\end{array}\right.
$$

Dong et al. [5] showed that the numerical scale model provides a unified framework to connect the 2-tuple linguistic model [10], the proportional 2-tuple linguistic model [36], and the unbalanced linguistic model [15]. To address the fact that words mean different things for different people, Li et al. [19] proposed numerical scale-based consistencydriven optimization models to derive the different decision makers' PISs. They also presented the linguistic GDM with the PISs framework shown in Fig. 1.

In Fig. $1, \mathrm{NS}^{k}$ is an ordered numerical scale on $S$ associated with decision maker $e_{k}(k=1,2, \ldots, m)$, and the value of $\mathrm{NS}^{k}\left(s_{i}\right)$ represents the individual semantics of decision maker $e_{k}$ on the term $s_{i}(i=0,1, \ldots, g)$. The optimization models to obtain the PISs of decision makers under different decision making environments were proposed in [20] and [21]. Without loss of generality, in this article, the decision makers' numerical scales range is set as $[0,1]$, instead of $\mathbb{R}$.

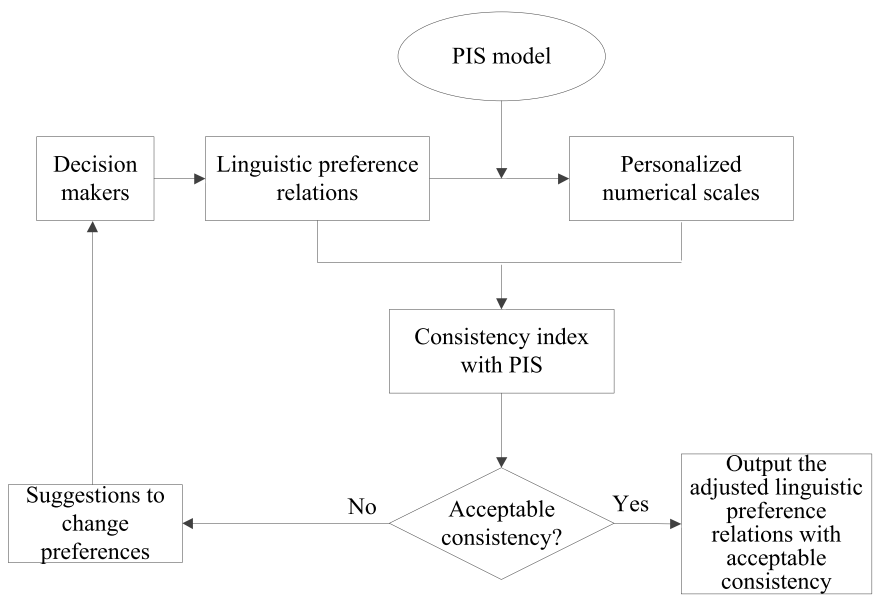

Fig. 2. Framework of the consistency improving process with PISs.

\section{Consistency IMProving ApProACH BASED ON PISs With Linguistic PREFERENCE RELATION}

This section presents a novel consistency index based on the personalized numerical scales for linguistic preference relations, and a consistency improving method with PISs in linguistic GDM.

\section{A. Description of the Decision Problem}

In the linguistic GDM, $X=\left\{x_{1}, x_{2}, \ldots, x_{n}\right\}(n \geq 2)$ denotes a set of alternatives and $E=\left\{e_{1}, e_{2}, \ldots, e_{m}\right\}(m \geq 2)$ denotes a set of decision makers, who express their preferences using linguistic terms in set $S=\left\{s_{0}, s_{1}, \ldots, s_{g}\right\}(g \geq 2)$ : $L^{k}=\left(l_{i j}^{k}\right)_{n \times n}$ denotes the linguistic preference relation over $X$ provided by decision maker $e_{k}$. Decision makers have their own, possibly different, personalized numerical scales over $S: \mathrm{NS}^{k}$ denotes the PIS of decision maker $e_{k}$. The problem to address is how to improve the consistency of a linguistic preference relation in GDM taking into account the decision maker's PIS.

Fig. 2 illustrates the three phases consistency improving framework with PISs.

1) PIS Process: A decision maker's PIS is obtained by solving the corresponding linguistic preference relation with the consistency-driven optimization model.

2) Consistency Measurements Based on the PISs: The consistency of linguistic preference relations with PISs is measured to judge whether their consistency is acceptable within the PIS context.

3) Feedback Recommendation for Improving Consistency: Decision makers with unacceptable consistency based on PIS values receive feedback on how to improve their linguistic preference relations' consistency.

\section{B. Consistency-Based PIS Model With Linguistic Preference Relations}

Additive transitivity is commonly used to define the consistency of preferences. The concept of additive consistent linguistic preference relation based on the numerical scale has been defined as follows. 
Definition 6 [16], [21]: A linguistic preference relation on a linguistic term set $S, L=\left(l_{i j}\right)_{n \times n}$, is a consistent based on a numerical scale, NS $: S \rightarrow[0,1]$, if $\mathrm{NS}\left(l_{i j}\right)+\mathrm{NS}\left(l_{j z}\right)-$ $\mathrm{NS}\left(l_{i z}\right)=0.5 \forall i, j, z=1,2, \ldots, n$.

The following definitions are introduced for measuring the consistency of linguistic preference relations.

Definition 7: The distance between consistent linguistic preference relations on a linguistic term set $S$ based on a numerical scale NS is computed as follows:

$$
d_{\mathrm{NS}}\left(L^{1}, L^{2}\right)=\frac{2}{n(n-1)} \sum_{i=1}^{n} \sum_{j=i+1}^{n}\left|\mathrm{NS}\left(l_{i j}^{1}\right)-\mathrm{NS}\left(l_{i j}^{2}\right)\right| \text {. }
$$

Definition 8: Let $M_{n}$ be the set of $n \times n$ consistent linguistic preference relations on a linguistic term set $S$ based on a numerical scale NS. The distance between a linguistic preference relation on a linguistic term set $S$ and set $M_{n}$ is

$$
d_{\mathrm{NS}}\left(L, M_{n}\right)=\min _{\bar{L} \in M_{n}} d_{\mathrm{NS}}(L, \bar{L}) .
$$

The proximity of a linguistic preference relation on a linguistic term set $S$ to the set $M_{n}$ is proposed as a measure of its consistency index $(\mathrm{CI})$

$$
\mathrm{CI}_{\mathrm{NS}}(L)=1-d_{\mathrm{NS}}\left(L, M_{n}\right) \text {. }
$$

The larger the value $\mathrm{CI}_{\mathrm{NS}}(L) \in[0,1]$, the better the consistency of $L$.

Proposition 1: The consistency index of a linguistic preference relation based on the numerical scale

$$
\mathrm{NS}\left(s_{i}\right)=\frac{1}{g} \cdot \Delta^{-1}\left(s_{i}\right)
$$

per (6) coincides with the consistency index of the 2-tuple linguistic model per (3).

Proof: Omitted.

In the following, the PISs of a decision maker in linguistic GDM are obtained by developing a consistency-driven optimization model with objective function

$$
\max \mathrm{CI}_{\mathrm{NS}^{k}}\left(L^{k}\right)=\max _{\bar{L}^{k} \in M_{n}} 1-d_{\mathrm{NS}^{k}}\left(L^{k}, \bar{L}^{k}\right)
$$

with $\bar{L}^{k}=\left(\bar{l}_{i j}^{k}\right)_{n \times n} \in M_{n}$ being a consistent linguistic preference relation on a linguistic term set $S$ based on a numerical scale $\mathrm{NS}^{k}$, that is

$$
\mathrm{NS}^{k}\left(\bar{l}_{i j}^{k}\right)+\mathrm{NS}^{k}\left(\bar{l}_{j z}^{k}\right)-\mathrm{NS}^{k}\left(\bar{l}_{i z}^{k}\right)=0.5 \forall i, j, z
$$

and $\bar{l}_{i j}^{k}=\operatorname{Neg}\left(\bar{l}_{j i}^{k}\right) \forall i, j$.

The range of the numerical scale $\mathrm{NS}^{k}$ for linguistic terms $s_{r}(r=0,1, \ldots, g)$ can be set as follows:

$$
\mathrm{NS}^{k}\left(s_{r}\right)\left\{\begin{array}{lll}
=0, & r & =0 \\
\in\left[\frac{r-1}{g}, \frac{r+1}{g}\right], & r=1,2, \ldots, g-1 ; r \neq \frac{g}{2} \\
=0.5, & r & =\frac{g}{2} \\
=1, & r & =g .
\end{array}\right.
$$

Note 2: The set of the range of the numerical scales does not influence the essence of the PIS model. The core of the PIS model is to discuss the distribution of the personalized numerical scale values of linguistic terms within the established range. The semantics of linguistic terms are often defined in the interval $[0,1]$ and, thus, in this study, we set the values of the numerical scale for the linguistic term in the interval $[0,1]$.

To make $\mathrm{NS}^{k}$ ordered, the following constraint value $\lambda$ between numerical scales is introduced:

$$
\mathrm{NS}^{k}\left(s_{r+1}\right)-\mathrm{NS}^{k}\left(s_{r}\right) \geq \lambda, \text { for } r=0,1, \ldots, g-1 .
$$

In this article, we set $\lambda=0.01$.

Based on (7)-(10), the following consistency-driven optimization model derives the PIS of decision maker $e_{k}$ :

$$
\begin{aligned}
& \left\{\begin{array}{l}
\max \mathrm{CI}_{\mathrm{NS}^{k}}\left(L^{k}\right)=1-\frac{2}{n(n-1)} \sum_{i=1}^{n} \sum_{j=i+1}^{n}\left|\mathrm{NS}^{k}\left(l_{i j}^{k}\right)-\mathrm{NS}^{k}\left(\bar{l}_{i j}^{k}\right)\right| \\
\operatorname{s.t.NS^{k}}\left(\bar{l}_{i j}^{k}\right)+\mathrm{NS}^{k}\left(\bar{l}_{j z}^{k}\right)-\mathrm{NS}^{k}\left(\bar{l}_{i z}^{k}\right)=0.5 \text { for } i, j, z=1,2, \ldots, n \\
\bar{l}_{i j}^{k} \in S \text { for } i, j=1,2, \ldots, n \\
\bar{l}_{i j}^{k}=\operatorname{Neg}\left(\bar{l}_{j i}^{k}\right) \text { for } i, j=1,2, \ldots, n \\
\operatorname{NS}^{k}\left(s_{0}\right)=0
\end{array}\right. \\
& \mathrm{NS}^{k}\left(s_{r}\right) \in\left[\frac{r-1}{g}, \frac{r+1}{g}\right], r=1,2, \ldots, g-1 ; r \neq \frac{g}{2} \\
& \mathrm{NS}^{k}\left(s_{\frac{g}{2}}\right)=0.5 \\
& \mathrm{NS}^{k}\left(s_{g}\right)=1 \\
& \mathrm{NS}^{k}\left(s_{r+1}\right)-\mathrm{NS}^{k}\left(s_{r}\right) \geq \lambda, r=0,1, \ldots, g-1 .
\end{aligned}
$$

In Model (11), $\mathrm{NS}^{k}\left(s_{r}\right)(r=0,1, \ldots, g)$ and $\bar{l}_{i j}^{k}(i, j=$ $1,2, \ldots, n)$ are decision variables. By solving Model (11), we can obtain the personalized numerical scales of linguistic terms for decision makers, that is, $\mathrm{NS}^{k}\left(s_{r}\right)(r=0,1, \ldots, g)$. In addition, we can also obtain the associated consistent linguistic preference relations associated with $L^{k}$, that is, $\bar{L}^{k}=\left(\bar{l}_{i j}^{k}\right)_{n \times n}$. The decision variable $\mathrm{NS}^{k}\left(\bar{l}_{i j}^{k}\right)(i, j=1,2, \ldots, n)$ with the associated consistent numerical preference relation $\bar{L}^{k}$ shows the difference between Model (11) and the existing PIS models [19], [20], [22].

By solving Model (11), the personalized numerical scales for the different decision makers based on their personal understanding of words for decision makers, as represented by their provided linguistic preference relations, are obtained.

Note 3: Model (11) can be easily transformed into a linear programming model and, thus, the Weierstrass theorem guarantees the existence of the optimal solution(s) in Model (11) because it has a closed bounded nonempty feasible region. There exists a two-stage general procedure [2] to deal with the case that multiple optimal solutions exist in linear programming models. This procedure can directly be applied in Model (11), and for details, see [2]. In this article, we focus on the consistency improvement of linguistic preference relations, which is an iterative process with a feedback recommendation. The obtained optimal solution(s) just provide a reference for decision makers to modify their preferences and, thus, the uniqueness of the solution is not the focus of our model.

Following the novel consistency index of linguistic preference relations based on PISs is now introduced.

Definition 9: Let $\mathrm{NS}^{k}$ and $L^{k}$ be defined as before, and $\bar{L}^{k}=\left(\bar{l}_{i j}^{k}\right)_{n \times n}$ be the consistent linguistic preference relation obtained from Model (11). The consistency index of $L^{k}$ based 
on the PIS is computed as

$$
\mathrm{CI}_{\mathrm{NS}^{k}}\left(L^{k}\right)=1-\frac{2}{n(n-1)} \sum_{i=1}^{n} \sum_{j=i+1}^{n}\left|\mathrm{NS}^{k}\left(l_{i j}^{k}\right)-\mathrm{NS}^{k}\left(\bar{l}_{i j}^{k}\right)\right| .
$$

A larger value of $\mathrm{CI}_{\mathrm{NS}^{k}}\left(L^{k}\right)$ indicates a better consistency of $L^{k}$. When $\mathrm{CI}_{\mathrm{NS}}\left(L^{k}\right)=1, L^{k}$ is fully consistent.

\section{PIS-Based Consistency Improving Algorithm}

Next, we describe in detail the algorithm to improve the consistency of linguistic preference relations with PISs.

1) PIS Process: Apply the optimization Model (11) to obtain the PIS of $L^{k},\left\{\mathrm{NS}^{k}\left(s_{0}\right), \mathrm{NS}^{k}\left(s_{1}\right), \ldots, \mathrm{NS}^{k}\left(s_{g}\right)\right\}$, and its consistency index $\mathrm{CI}_{\mathrm{NS}^{k}}\left(L^{k}\right)$.

2) Feedback Recommendation for Improving Consistency: Let $\bar{L}^{k}=\left(\bar{l}_{i j}^{k}\right)_{n \times n}$, obtained from Model (11), be the consistent linguistic preference relation associated with $L^{k}$. A new linguistic preference relation $L^{\prime k}=\left(l_{i j}^{\prime k}\right)_{n \times n}$ is constructed based on $L^{k}$ and $\bar{L}^{k}$.

1) When $i_{i j}^{k}<\bar{l}_{i j}^{k}$, the decision maker $e_{k}$ should increase the preference value $l_{i j}^{k}$ to be closer to $\bar{l}_{i j}^{k}$, that is, $l_{i j}^{k} \in\left(l_{i j}^{k}, \bar{l}_{i j}^{k}\right]$.

2) When $l_{i j}^{k}>\bar{l}_{i j}^{k}$, the decision maker $e_{k}$ should decrease the preference value $l_{i j}^{k}$ to be closer to $\bar{l}_{i j}^{k}$, that is, $l_{i j}^{k} \in\left[\bar{l}_{i j}^{k}, l_{i j}^{k}\right)$.

3) When $l_{i j}^{k}=\bar{l}_{i j}^{k}$, then $e_{k}$ should not change the preference value $l_{i j}^{k}$, that is, $l_{i j}^{k}=l_{i j}^{k}=\bar{l}_{i j}^{k}$.

The PIS-based consistency improving algorithm is summarized in Algorithm 1.

The below results prove that Algorithm 1 increases the consistency index values.

Theorem 1: Let $\overline{\mathrm{CI}}$ be the consistency threshold in Algorithm 1. Let $L^{k, t}=\left(l_{i j}^{k, t}\right)_{n \times n}$ be the linguistic preference relations generated by Algorithm 1 and $\mathrm{CI}_{\mathrm{NS}^{k, t}}\left(L^{k, t}\right)$ its consistency index. Then, $\mathrm{CI}_{\mathrm{NS}^{k, t}}\left(L^{k, t}\right) \geq \overline{\mathrm{CI}} \forall k$; otherwise, if $\exists k$ : $\mathrm{CI}_{\mathrm{NS}^{k, t}}\left(L^{k, t}\right)<\overline{\mathrm{CI}}$, then $\mathrm{CI}_{\mathrm{NS}^{k, t}}\left(L^{k, t}\right)$ is monotone increasing, with respect to $t$, toward $\overline{\mathrm{CI}}$.

Proof: In Algorithm 1, by solving Model (11), we obtain the consistency index of $L^{k, t}: \mathrm{CI}_{\mathrm{NS}^{k, t}}\left(L^{k, t}\right)$. If $\exists k$ : $\mathrm{CI}_{\mathrm{NS}^{k, t}}\left(L^{k, t}\right)<\overline{\mathrm{CI}}$, then a consistent linguistic preference relation $\bar{L}^{k, t}$ associated to $L^{k, t}$, is constructed: $\mathrm{CI}_{\mathrm{NS}^{k, t}}\left(\bar{L}^{k, t}\right)=1$. Based on (13)

$$
\begin{aligned}
l_{i j}^{k, t+1} \in & {\left[\min \left(l_{i j}^{k, t}, \bar{l}_{i j}^{k, t}\right), \max \left(l_{i j}^{k, t}, \bar{l}_{i j}^{k, t}\right)\right] \Longrightarrow \operatorname{NS}\left(l_{i j}^{k, t+1}\right) } \\
& \times \in\left[\operatorname{NS}\left(\min \left(l_{i j}^{k, t}, \bar{l}_{i j}^{k, t}\right)\right), \operatorname{NS}\left(\max \left(l_{i j}^{k, t}, \bar{l}_{i j}^{k, t}\right)\right)\right] \\
d\left(l_{i j}^{k, t}, \bar{l}_{i j}^{k, t}\right) \geq & d\left(l_{i j}^{k, t+1}, \bar{l}_{i j}^{k, t}\right) \geq d\left(l_{i j}^{k, t+1}, \bar{l}_{i j}^{k, t+1}\right) \quad \forall i, j .
\end{aligned}
$$

From Definition 7, it is

$$
\begin{aligned}
d_{\mathrm{NS}}\left(L^{k, t}, \bar{L}^{k, t}\right) \geq & d_{\mathrm{NS}}\left(L^{k, t+1}, \bar{L}^{k, t}\right) \geq d_{\mathrm{NS}}\left(L^{k, t+1}, \bar{L}^{k, t+1}\right) \\
& \Longrightarrow \mathrm{CI}_{\mathrm{NS}^{k, t}}\left(L^{k, t}\right) \leq \mathrm{CI}_{\mathrm{NS}^{k, t+1}}\left(L^{k, t+1}\right) .
\end{aligned}
$$

The sequence $\left\{\mathrm{CI}_{\mathrm{NS}^{k, t}}\left(L^{k, t}\right) \mid t=0,1,2, \ldots, T\right\}$ is monotone increasing toward $\overline{\mathrm{CI}}$.
Algorithm 1 PIS-Based Consistency Improving Algorithm

Input: The linguistic term set $S=\left\{s_{0}, s_{1}, \ldots, s_{g}\right\}$; the set of decision makers $E=\left\{e_{1}, e_{2}, \ldots, e_{m}\right\}$; the linguistic preference relations $\left\{L^{k}=\left(l_{i j}^{k}\right)_{n \times n} \mid k=1, \ldots, m\right\}$; the consistency threshold $\overline{C I}$; and the maximum number of iterations $T$.

Output: The adjusted linguistic preference relations $\left\{L^{\prime k}=\left(l_{i j}^{k}\right)_{n \times n} \mid k=1, \ldots, m\right\}$ and their consistency indices $\left\{C I_{N S^{k, t}}\left(L^{\prime} k\right) \mid k=1, \ldots, m\right\}$.

Step 1: Let $t=0$, and $L^{k, t}=\left(l_{i j}^{k, t}\right)_{n \times n}=L^{k, 0}=\left(l_{i j}^{k}\right)_{n \times n}$.

Step 2: Solve Model (11) to obtain the PISs of $\left\{L^{k, t} \mid k=1, \ldots, m\right\}, \quad\left\{N S^{k, t} \quad\left(s_{0}\right), \quad N S^{k, t} \quad\left(s_{1}\right), \quad \ldots, \quad N S^{k, t}\right.$ $\left.\left(s_{g}\right) \mid k=1, \ldots, m\right\}$, the associated consistent linguistic preference relation $\bar{L}^{k, t}=\left(\bar{l}_{i j}^{k, t}\right)_{n \times n}$ with $\bar{l}_{i j}^{k, t}=N S^{-1, k}\left(N S^{k, t}\left(\bar{l}_{i j}^{k, t}\right)\right)$, and their consistency indices $\left\{C I_{N S^{k, t}}\left(L^{k, t}\right) \mid k=1, \ldots, m\right\}$. If $C I_{N S^{k, t}}\left(L^{k, t}\right) \geq \overline{C I} \forall k$ or $t=T$, then go to Step 4; otherwise, go to Step 3 .

Step 3: Based on $\bar{L}^{k, t}=\left(\bar{l}_{i j}^{k, t}\right)_{n * n}$, to obtain $L^{k, t+1}=\left(l_{i j}^{k, t+1}\right)_{n \times n}$, it is required that,

$$
l_{i j}^{k, t+1} \begin{cases}\in\left(l_{i j}^{k, t}, \bar{l}_{i j}^{k, t}\right], & \text { If } l_{i j}^{k, t}<\bar{l}_{i j}^{k, t} \\ \in\left[\bar{l}_{i j}^{k, t}, l_{i j}^{k, t}\right), & \text { If } l_{i j}^{k, t}>\bar{l}_{i j}^{k, t} \\ =l_{i j}^{k, t}, & \text { If } l_{i j}^{k, t}=\bar{l}_{i j}^{k, t}\end{cases}
$$

Let $t=t+1$, return to Step 2 .

Step 4: Let $L^{\prime} k=L^{k, t}$. Output the adjusted linguistic preference relation with acceptable consistency $\left\{L^{\prime k}=\left(l_{i j}^{\prime}\right)_{n \times n} \mid k=1, \ldots, m\right\}$ and their consistency indices $\left\{C I_{N S^{k, t}}\left(L^{\prime k}\right) \mid k=1, \ldots, m\right\}$.

Theorem 1 guarantees that the adjusted linguistic preference relations obtained by the PIS-based consistency improving algorithm (Algorithm 1) will have the acceptable consistency or a higher consistency degree close to the threshold value $\overline{\mathrm{CI}}$.

Note 4: The value of $\overline{\mathrm{CI}}$ is to determine whether the consistency of a linguistic preference relation is reached. The value of $\overline{\mathrm{CI}}$ is different to different decision-making problems, and it should be set according to the specific decision-making contexts. While Algorithm 1 provides a general approach to improve the consistency of linguistic preference relations based on PISs, and it works when setting different threshold values $\overline{\mathrm{CI}}$.

\section{NumERICAL ANALYSiS}

In this section, numerical examples are included to illustrate the use of the consistency improving algorithm with PISs using the linguistic term set $S=\left\{s_{0}=\right.$ extremely poor, $s_{1}=$ very poor, $s_{2}=$ poor, $s_{3}=$ fair, $s_{4}=$ good, $s_{5}=$ very good, $s_{6}=$ extremely good $\}$, a set of four decision makers, $E=\left\{e_{1}, e_{2}, e_{3}, e_{4}\right\}$, and a set of five alternatives, $X=\left\{x_{1}, x_{2}, x_{3}, x_{4}, x_{5}\right\}$. The decision makers provide the below linguistic preference relations based on $S, L^{k}=\left(l_{i j}^{k}\right)_{5 \times 5}(k=$ 
TABLE I

VALUES OF NS ${ }^{k, 0}\left(s_{i}\right)(k=1,2,3,4 ; i=0,1, \ldots, 6)$

\begin{tabular}{l|c|c|c|c}
\hline & $k=1$ & $k=2$ & $k=3$ & $k=4$ \\
\hline$N S^{k, 1}\left(s_{0}\right)$ & 0 & 0 & 0 & 0 \\
\hline$N S^{k, 1}\left(s_{1}\right)$ & 0.333 & 0.01 & 0.333 & 0.333 \\
\hline$N S^{k, 1}\left(s_{2}\right)$ & 0.49 & 0.49 & 0.49 & 0.343 \\
\hline$N S^{k, 1}\left(s_{3}\right)$ & 0.5 & 0.5 & 0.5 & 0.5 \\
\hline$N S^{k, 1}\left(s_{4}\right)$ & 0.657 & 0.51 & 0.51 & 0.657 \\
\hline$N S^{k, 1}\left(s_{5}\right)$ & 0.667 & 0.667 & 0.667 & 0.667 \\
\hline$N S^{k, 1}\left(s_{6}\right)$ & 1 & 1 & 1 & 1 \\
\hline
\end{tabular}

$1,2,3,4)$, to express their preferences over $X$

$$
\begin{aligned}
L^{1} & =\left(\begin{array}{ccccc}
\text { null } & s_{4} & s_{1} & s_{6} & s_{5} \\
\text { null } & \text { null } & s_{2} & s_{3} & s_{3} \\
\text { null } & \text { null } & \text { null } & s_{0} & s_{5} \\
\text { null } & \text { null } & \text { null } & \text { null } & s_{2} \\
\text { null } & \text { null } & \text { null } & \text { null } & \text { null }
\end{array}\right) \\
L^{2} & =\left(\begin{array}{llccc}
\text { null } & s_{6} & s_{4} & s_{0} & s_{0} \\
\text { null } & \text { null } & s_{6} & s_{3} & s_{2} \\
\text { null } & \text { null } & \text { null } & s_{5} & s_{1} \\
\text { null } & \text { null } & \text { null } & \text { null } & s_{6} \\
\text { null } & \text { null } & \text { null } & \text { null } & \text { null }
\end{array}\right) \\
L^{3} & =\left(\begin{array}{llccc}
\text { null } & s_{0} & s_{6} & s_{0} & s_{4} \\
\text { null } & \text { null } & s_{1} & s_{5} & s_{0} \\
\text { null } & \text { null } & \text { null } & s_{6} & s_{2} \\
\text { null } & \text { null } & \text { null } & \text { null } & s_{5} \\
\text { null } & \text { null } & \text { null } & \text { null } & \text { null }
\end{array}\right) \\
L^{4}= & \left(\begin{array}{lllll}
\text { null } & s_{1} & s_{6} & s_{6} & s_{0} \\
\text { null } & \text { null } & s_{0} & s_{5} & s_{1} \\
\text { null } & \text { null } & \text { null } & s_{4} & s_{2} \\
\text { null } & \text { null } & \text { null } & \text { null } & s_{6} \\
\text { null } & \text { null } & \text { null } & \text { null } & \text { null }
\end{array}\right) .
\end{aligned}
$$

\section{A. First Iteration With PISs}

Let $L^{1}=L^{1,0}, L^{2}=L^{2,0}, L^{3}=L^{3,0}$, and $L^{4}=L^{4,0}$. Solving Model (11) with linguistic preference relations $L^{k, 0}(k=$ $1,2,3,4)$, the PISs for the linguistic terms for the four decision makers, $\mathrm{NS}^{k, 0}\left(s_{i}\right)(k=1,2,3,4 ; i=0,1, \ldots, 6)$, are obtained, and listed in Table I.

The consistency indices based on the PISs are

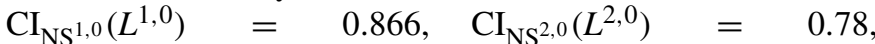
$\mathrm{CI}_{\mathrm{NS}^{3,0}}\left(L^{3,0}\right)=0.698$, and $\mathrm{CI}_{\mathrm{NS}^{4,0}}\left(L^{4,0}\right)=0.731$.

And from Model (11), it also obtains the associated consistent linguistic preference relations $\bar{L}^{k, 0}(k=1,2,3,4)$ as follows:

$\bar{L}^{1,0}=\left(\begin{array}{ccccc}\text { null } & \left(s_{5},-0.4\right) & \left(s_{3},-0.261\right) & s_{5} & \left(s_{5}, 0.057\right) \\ \text { null } & \text { null } & \left(s_{1}, 0.287\right) & \left(s_{3}, 0.025\right) & \left(s_{3}, 0.146\right) \\ \text { null } & \text { null } & \text { null } & \left(s_{4},-0.204\right) & \left(s_{4},-0.083\right) \\ \text { null } & \text { null } & \text { null } & \text { null } & \left(s_{3}, 0.121\right) \\ \text { null } & \text { null } & \text { null } & \text { null } & \text { null }\end{array}\right)$

$$
\begin{aligned}
\bar{L}^{2,0} & =\left(\begin{array}{llccc}
\text { null } & \left(s_{1}, 0.053\right) & s_{4} & \left(s_{1}, 0.053\right) & \left(s_{1}, 0.026\right) \\
\text { null } & \text { null } & \left(s_{6},-0.06\right) & s_{3} & s_{2} \\
\text { null } & \text { null } & \text { null } & \left(s_{1}, 0.026\right) & s_{1} \\
\text { null } & \text { null } & \text { null } & \text { null } & s_{2} \\
\text { null } & \text { null } & \text { null } & \text { null } & \text { null }
\end{array}\right) \\
\bar{L}^{3,0} & =\left(\begin{array}{llcccc}
\text { null } & \left(s_{2},-0.401\right) & \left(s_{2},-0.083\right) & \left(s_{1}, 0.452\right) & \left(s_{1}, 0.038\right) \\
\text { null } & \text { null } & \left(s_{4}, 0.025\right) & \left(s_{2},-0.083\right) & \left(s_{4}, 0.299\right) \\
\text { null } & \text { null } & \text { null } & \left(s_{2},-0.401\right) & \left(s_{4},-0.3\right) \\
\text { null } & \text { null } & \text { null } & \text { null } & \left(s_{4}, 0.446\right) \\
\text { null } & \text { null } & \text { null } & \text { null } & \text { null }
\end{array}\right) \\
\bar{L}^{4,0} & =\left(\begin{array}{llcccc}
\text { null } & \left(s_{6},-0.441\right) & \left(s_{6},-0.471\right) & s_{6} & \left(s_{5}, 0.057\right) \\
\text { null } & \text { null } & \left(s_{3},-0.064\right) & \left(s_{4},-0.064\right) & s_{1} \\
\text { null } & \text { null } & \text { null } & s_{4} & s_{2} \\
\text { null } & \text { null } & \text { null } & \text { null } & \left(s_{1},-0.442\right) \\
\text { null } & \text { null } & \text { null } & \text { null } & \text { null }
\end{array}\right) .
\end{aligned}
$$

Then, the adjusted linguistic preference relations, $L^{k, 1}(k=$ $1,2,3,4)$, that satisfy $l_{i j}^{k, 1} \in\left[\min \left(l_{i j}^{k, 0}, \bar{l}_{i j}^{k, 0}\right), \max \left(l_{i j}^{k, 0}, \bar{l}_{i j}^{k, 0}\right)\right]$ and $l_{i j}^{k, 1} \neq l_{i j}^{k, 0}$, are

$$
\begin{aligned}
L^{1,1}= & \left(\begin{array}{ccccc}
\text { null } & s_{4} & s_{2} & s_{5} & s_{5} \\
\text { null } & \text { null } & s_{2} & s_{3} & s_{3} \\
\text { null } & \text { null } & \text { null } & s_{2} & s_{4} \\
\text { null } & \text { null } & \text { null } & \text { null } & s_{3} \\
\text { null } & \text { null } & \text { null } & \text { null } & \text { null }
\end{array}\right) \\
L^{2,1}= & \left(\begin{array}{ccccc}
\text { null } & s_{4} & s_{4} & s_{0} & s_{1} \\
\text { null } & \text { null } & s_{6} & s_{3} & s_{2} \\
\text { null } & \text { null } & \text { null } & s_{2} & s_{1} \\
\text { null } & \text { null } & \text { null } & \text { null } & s_{5} \\
\text { null } & \text { null } & \text { null } & \text { null } & \text { null }
\end{array}\right) \\
L^{3,1}= & \left.\begin{array}{lcccc}
\text { null } & s_{1} & s_{5} & s_{1} & s_{3} \\
\text { null } & \text { null } & s_{2} & s_{4} & s_{1} \\
\text { null } & \text { null } & \text { null } & s_{5} & s_{3} \\
\text { null } & \text { null } & \text { null } & \text { null } & s_{4} \\
\text { null } & \text { null } & \text { null } & \text { null } & \text { null }
\end{array}\right) \\
L^{4,1}= & \left.\begin{array}{lllll}
\text { null } & s_{5} & s_{6} & s_{6} & s_{3} \\
\text { null } & \text { null } & s_{2} & s_{4} & s_{1} \\
\text { null } & \text { null } & \text { null } & s_{4} & s_{2} \\
\text { null } & \text { null } & \text { null } & \text { null } & s_{3} \\
\text { null } & \text { null } & \text { null } & \text { null } & \text { null }
\end{array}\right) .
\end{aligned}
$$

By solving Model (11) with linguistic preference relations $L^{1,1}, L^{2,1}, \mathrm{~L}^{3,1}$, and $L^{4,1}$, the PISs for linguistic terms for the four decision makers, $\mathrm{NS}^{k, 1}\left(s_{i}\right)(k=1,2,3,4 ; i=0,1, \ldots, 6)$, are obtained and listed in Table II.

The consistency indices based on the PISs are $\mathrm{CI}_{\mathrm{NS}^{1,1}}\left(L^{1,1}\right)=0.965, \mathrm{CI}_{\mathrm{NS}^{2,1}}\left(L^{2,1}\right)=0.881, \mathrm{CI}_{\mathrm{NS}^{3,1}}\left(L^{3,1}\right)=$ 0.913 , and $\mathrm{CI}_{\mathrm{NS}^{4,1}}\left(L^{4,1}\right)=0.9$.

\section{B. Second Iteration With PISs}

By solving Model (11), we also obtain the associated consistent linguistic preference relations, $\bar{L}^{k, 1}(k=1,2,3,4)$, as 
TABLE II

VALUES OF $\mathrm{NS}^{k, 1}\left(s_{i}\right)(k=1,2,3,4 ; i=0,1, \ldots, 6)$

\begin{tabular}{l|c|c|c|c}
\hline & $k=1$ & $k=2$ & $k=3$ & $k=4$ \\
\hline$N S^{k, 1}\left(s_{0}\right)$ & 0 & 0 & 0 & 0 \\
\hline$N S^{k, 1}\left(s_{1}\right)$ & 0.1 & 0.01 & 0.333 & 0.333 \\
\hline$N S^{k, 1}\left(s_{2}\right)$ & 0.49 & 0.196 & 0.49 & 0.49 \\
\hline$N S^{k, 1}\left(s_{3}\right)$ & 0.5 & 0.5 & 0.5 & 0.5 \\
\hline$N S^{k, 1}\left(s_{4}\right)$ & 0.604 & 0.51 & 0.51 & 0.51 \\
\hline$N S^{k, 1}\left(s_{5}\right)$ & 0.667 & 0.667 & 0.667 & 0.99 \\
\hline$N S^{k, 1}\left(s_{6}\right)$ & 1 & 1 & 1 & 1 \\
\hline
\end{tabular}

follows:

$\bar{L}^{1,1}=\left(\begin{array}{ccccc}\text { null } & \left(s_{4}, 0.465\right) & \left(s_{4},-0.307\right) & \left(s_{4}, 0.365\right) & \left(s_{5},-0.19\right) \\ \text { null } & \text { null } & \left(s_{2},-0.11\right) & \left(s_{3}, 0.125\right) & \left(s_{3}, 0.298\right) \\ \text { null } & \text { null } & \text { null } & \left(s_{4},-0.375\right) & \left(s_{4},-0.202\right) \\ \text { null } & \text { null } & \text { null } & \text { null } & \left(s_{3}, 0.173\right) \\ \text { null } & \text { null } & \text { null } & \text { null } & \text { null }\end{array}\right)$

$\bar{L}^{2,1}=\left(\begin{array}{ccccc}\text { null } & \left(s_{2}, 0.076\right) & s_{4} & s_{1} & \left(s_{1}, 0.027\right) \\ \text { null } & \text { null } & \left(s_{5}, 0.372\right) & \left(s_{2}, 0.3125\right) & \left(s_{2}, 0.329\right) \\ \text { null } & \text { null } & \text { null } & s_{0} & s_{0} \\ \text { null } & \text { null } & \text { null } & \text { null } & \left(s_{4},-0.5\right) \\ \text { null } & \text { null } & \text { null } & \text { null } & \text { null }\end{array}\right)$

$\bar{L}^{3,1}=\left(\begin{array}{ccccc}\text { null } & \left(s_{2},-0.452\right) & \left(s_{2},-0.401\right) & \left(s_{1},-0.42\right) & \left(s_{2},-0.395\right) \\ \text { null } & \text { null } & \left(s_{4},-0.1\right) & \left(s_{4},-0.4\right) & \left(s_{4},-0.1\right) \\ \text { null } & \text { null } & \text { null } & \left(s_{4},-0.3\right) & s_{3} \\ \text { null } & \text { null } & \text { null } & \text { null } & s_{3} \\ \text { null } & \text { null } & \text { null } & \text { null } & \text { null }\end{array}\right)$

$\bar{L}^{4,1}=\left(\begin{array}{ccccc}\text { null } & \left(s_{6},-0.3\right) & \left(s_{5},-0.04\right) & \left(s_{6},-0.1\right) & \left(s_{5},-0.144\right) \\ \text { null } & \text { null } & \left(s_{2}, 0.1\right) & \left(s_{3}, 0.2\right) & \left(s_{2},-0.42\right) \\ \text { null } & \text { null } & \text { null } & \left(s_{4}, 0.02\right) & \left(s_{2},-0.363\right) \\ \text { null } & \text { null } & \text { null } & \text { null } & \left(s_{2},-0.433\right) \\ \text { null } & \text { null } & \text { null } & \text { null } & \text { null }\end{array}\right)$.

The adjusted linguistic preference relation, $L^{k, 2}(k=1,2,3,4)$, that satisfy $l_{i j}^{k, 2} \in\left(l_{i j}^{k, 1}, \bar{l}_{i j}^{k, 1}\right]$ are

$$
\begin{aligned}
L^{1,2} & =\left(\begin{array}{ccccc}
\text { null } & s_{4} & s_{3} & s_{5} & s_{5} \\
\text { null } & \text { null } & s_{2} & s_{3} & s_{3} \\
\text { null } & \text { null } & \text { null } & s_{3} & s_{4} \\
\text { null } & \text { null } & \text { null } & \text { null } & s_{3} \\
\text { null } & \text { null } & \text { null } & \text { null } & \text { null }
\end{array}\right) \\
L^{2,2} & =\left(\begin{array}{ccccc}
\text { null } & s_{3} & s_{4} & s_{1} & s_{1} \\
\text { null } & \text { null } & s_{6} & s_{3} & s_{2} \\
\text { null } & \text { null } & \text { null } & s_{0} & s_{0} \\
\text { null } & \text { null } & \text { null } & \text { null } & s_{4} \\
\text { null } & \text { null } & \text { null } & \text { null } & \text { null }
\end{array}\right) \\
L^{3,2} & =\left(\begin{array}{lcccc}
\text { null } & s_{1} & s_{2} & s_{1} & s_{2} \\
\text { null } & \text { null } & s_{3} & s_{4} & s_{3} \\
\text { null } & \text { null } & \text { null } & s_{4} & s_{3} \\
\text { null } & \text { null } & \text { null } & \text { null } & s_{3} \\
\text { null } & \text { null } & \text { null } & \text { null } & \text { null }
\end{array}\right)
\end{aligned}
$$

TABLE III

VALUES OF $\operatorname{NS}^{k, 2}\left(s_{i}\right)(k=1,2,3,4 ; i=0,1, \ldots, 6)$

\begin{tabular}{l|c|c|c|c}
\hline & $k=1$ & $k=2$ & $k=3$ & $k=4$ \\
\hline$N S^{k, 2}\left(s_{0}\right)$ & 0 & 0 & 0 & 0 \\
\hline$N S^{k, 2}\left(s_{1}\right)$ & 0.1 & 0.333 & 0.157 & 0.333 \\
\hline$N S^{k, 2}\left(s_{2}\right)$ & 0.343 & 0.49 & 0.167 & 0.343 \\
\hline$N S^{k, 2}\left(s_{3}\right)$ & 0.5 & 0.5 & 0.5 & 0.5 \\
\hline$N S^{k, 2}\left(s_{4}\right)$ & 0.657 & 0.51 & 0.51 & 0.671 \\
\hline$N S^{k, 2}\left(s_{5}\right)$ & 0.667 & 0.828 & 0.667 & 0.828 \\
\hline$N S^{k, 2}\left(s_{6}\right)$ & 1 & 1 & 1 & 1 \\
\hline
\end{tabular}

$$
L^{4,2}=\left(\begin{array}{ccccc}
\text { null } & s_{5} & s_{5} & s_{6} & s_{4} \\
\text { null } & \text { null } & s_{2} & s_{4} & s_{1} \\
\text { null } & \text { null } & \text { null } & s_{4} & s_{2} \\
\text { null } & \text { null } & \text { null } & \text { null } & s_{2} \\
\text { null } & \text { null } & \text { null } & \text { null } & \text { null }
\end{array}\right)
$$

The PISs of $L^{k, 2}(k=1,2,3,4)$ are obtained and listed in Table III.

The consistency indices based on the PISs are

$$
\begin{aligned}
& \mathrm{CI}_{\mathrm{NS}^{1,2}}\left(L^{1,2}\right)=0.983, \mathrm{CI}_{\mathrm{NS}^{2,2}}\left(L^{2,2}\right)=0.949 \\
& \mathrm{CI}_{\mathrm{NS}^{3,2}}\left(L^{3,2}\right)=0.996, \text { and } \mathrm{CI}_{\mathrm{NS}^{4,2}}\left(L^{4,2}\right)=0.966 .
\end{aligned}
$$

In accordance with Theorem 1, the numerical analysis clearly corroborates that the consistency indices of the linguistic preference relations increase in value from one round application of Algorithm 1 to the next.

\section{Comparative Study}

This section reports on a comparative study between the PIS-based consistency improving method (Algorithm 1) and the corresponding one without implementation, which is based on the 2-tuple linguistic model (Algorithm 2).

\section{A. Consistency Improving Method Without PISs}

When PISs have no role, decision makers are assumed to have the same words' semantics, and the 2-tuple linguistic model is used as the linguistic computational model. Algorithm 2 derives from Algorithm 1 by replacing all the NSs with the function $\Delta^{-1}$ in the representation of the semantics of linguistic expressions, that is, we set $\mathrm{NS}^{k}\left(s_{i}\right)=\Delta^{-1}\left(s_{i}\right)$ for linguistic terms $s_{i}(i=0,1, \ldots, g)$ for decision makers $e_{k}(k=1,2, \ldots, m)$.

We apply Algorithm 2 to the same linguistic preference relations $L^{k}(k=1,2,3,4)$ provided in Section IV. The semantics of linguistic terms $\left\{s_{0}, s_{1}, \ldots, s_{6}\right\}$ based on the 2-tuple linguistic model for all decision makers are $\Delta^{-1}\left(s_{0}\right)=0$; $\Delta^{-1}\left(s_{1}\right)=0.167 ; \Delta^{-1}\left(s_{2}\right)=0.333 ; \Delta^{-1}\left(s_{3}\right)=0.5$; $\Delta^{-1}\left(s_{4}\right)=0.667 ; \Delta^{-1}\left(s_{5}\right)=0.833 ;$ and $\Delta^{-1}\left(s_{6}\right)=1$.

1) First Iteration Without Considering PISs: The linguistic preference relations are transformed into their associated 
$\overline{\text { Algorithm } 2 \text { Consistency Improving Algorithm Based on the }}$ 2-Tuple Linguistic Model

Input: The linguistic term set $S=\left\{s_{0}, s_{1}, \ldots, s_{g}\right\}$; the set of decision makers $E=\left\{e_{1}, e_{2}, \ldots, e_{m}\right\}$; the linguistic preference relations $\left\{L^{k}=\left(l_{i j}^{k}\right)_{n \times n} \mid k=1, \ldots, m\right\}$; the consistency threshold $\overline{C I}$; and the maximum number of iterations $T$.

Output: The adjusted linguistic preference relations $\left\{L^{\prime k}=\left(l_{i j}^{k}\right)_{n \times n} \mid k=1, \ldots, m\right\}$ and their consistency indices $\left\{C I\left(L^{\prime}\right) \mid k=1, \ldots, m\right\}$.

Step 1: Let $t=0$, let $L^{k, t}=\left(l_{i j}^{k, t}\right)_{n \times n}=L^{k, 0}=\left(l_{i j}^{k}\right)_{n \times n}$.

Step 2: Construct the associated numerical preference relation of $L^{k, t}, F^{k, t}=\left(f_{i j}^{k, t}\right)_{n \times n}$, where $f_{i j}^{k, t}=\Delta^{-1}\left(l_{i j}^{k, t}\right)$. If $C I\left(F^{k, t}\right) \geq \overline{C I}$ or $t=T$, then go to Step 5; otherwise, go to Step 3 .

Step 3: If $C I\left(F^{k, t}\right)$ is unacceptable, then construct the consistent numerical preference relation $\bar{F}^{k, t}=\left(\bar{f}_{i j}^{k, t}\right)_{n \times n}$ associated to $F^{k}$ by solving the following model:

$$
\left\{\begin{array}{l}
\min d\left(F^{k, t}, \bar{F}^{k, t}\right) \\
\text { s.t. } \\
\bar{f}_{i j}^{k, t}+\bar{f}_{j z}^{k, t}-\bar{f}_{i z}^{k, t}=0.5 \text { for } i, j, z=1,2, \ldots, n \\
\bar{f}_{i j}^{k, t} \in[0,1] \text { for } i, j=1,2, \ldots, n \\
\bar{f}_{i j}^{k, t}+\bar{f}_{j i}^{k, t}=1 \text { for } i, j=1,2, \ldots, n
\end{array}\right.
$$

where $d\left(F^{k, t}, \bar{F}^{k, t}\right)=\frac{2}{n(n-1)} \sum_{i=1}^{n} \sum_{j=i+1}^{n}\left|f_{i j}^{k, t}-\bar{f}_{i j}^{k, t}\right|$. Solving Model (14) obtains the consistent numerical preference relation $\bar{F}^{k, t}=\left(\bar{f}_{i j}^{k, t}\right)_{n \times n}$ associated to $F^{k, t}$.

Step 4: Construct the associated linguistic preference relation $\bar{L}^{k, t}=\left(\bar{l}_{i j}^{k, t}\right)_{n \times n}$ of $\bar{F}^{k, t}$, where $\bar{l}_{i j}^{k, t}=\Delta\left(\bar{f}_{i j}^{k, t}\right)$. For $L^{k, t+1}=\left(l_{i j}^{k, t+1}\right)_{n \times n}$, it is required that

$$
l_{i j}^{k, t+1} \begin{cases}\in\left(l_{i j}^{k, t}, \bar{l}_{i j}^{k, t}\right], & \text { If } l_{i j}^{k, t}<\bar{l}_{i j}^{k, t} \\ \in\left[\bar{l}_{i j}^{k, t}, l_{i j}^{k, t}\right), & \text { If } l_{i j}^{k, t}>\bar{l}_{i j}^{k, t} \\ =l_{i j}^{k, t}, & \text { If } l_{i j}^{k, t}=\bar{l}_{i j}^{k, t}\end{cases}
$$

Let $t=t+1$, return to Step 2 .

Step 5: Let $L^{\prime k}=L^{k, t}$. Output the adjusted linguistic preference relation with acceptable consistency $\left\{L^{\prime k}=\left(l_{i j}^{\prime}\right)_{n \times n} \mid k=1, \ldots, m\right\}$ and their consistency indices $\left\{C I\left(L^{\prime} k\right) \mid k=1, \ldots, m\right\}$.

numerical ones

$$
\begin{aligned}
F^{1,0} & =\left(\begin{array}{ccccc}
0.5 & 0.667 & 0.167 & 1 & 0.833 \\
\text { null } & 0.5 & 0.333 & 0.5 & 0.5 \\
\text { null } & \text { null } & 0.5 & 0 & 0.833 \\
\text { null } & \text { null } & \text { null } & 0.5 & 0.333 \\
\text { null } & \text { null } & \text { null } & \text { null } & 0.5
\end{array}\right) \\
F^{2,0} & =\left(\begin{array}{ccccc}
0.5 & 1 & 0.667 & 0 & 0 \\
\text { null } & 0.5 & 1 & 0.5 & 0.333 \\
\text { null } & \text { null } & 0.5 & 0.833 & 0.167 \\
\text { null } & \text { null } & \text { null } & 0.5 & 1 \\
\text { null } & \text { null } & \text { null } & \text { null } & 0.5
\end{array}\right) \\
F^{3,0} & =\left(\begin{array}{ccccc}
0.5 & 0 & 1 & 0 & 0.667 \\
\text { null } & 0.5 & 0.167 & 0.833 & 0 \\
\text { null } & \text { null } & 0.5 & 1 & 0.333 \\
\text { null } & \text { null } & \text { null } & 0.5 & 0.833 \\
\text { null } & \text { null } & \text { null } & \text { null } & 0.5
\end{array}\right)
\end{aligned}
$$

$$
F^{4,0}=\left(\begin{array}{ccccc}
0.5 & 0.167 & 1 & 1 & 0 \\
\text { null } & 0.5 & 0 & 0.833 & 0.167 \\
\text { null } & \text { null } & 0.5 & 0.667 & 0.333 \\
\text { null } & \text { null } & \text { null } & 0.5 & 1 \\
\text { null } & \text { null } & \text { null } & \text { null } & 0.5
\end{array}\right)
$$

By solving Model (14), the consistent numerical preference relations are

$$
\begin{aligned}
\bar{F}^{1,0} & =\left(\begin{array}{ccccc}
0.5 & 0.729 & 0.56 & 0.9 & 0.733 \\
0.271 & 0.5 & 0.331 & 0.67 & 0.503 \\
0.44 & 0.669 & 0.5 & 0.84 & 0.672 \\
0.1 & 0.33 & 0.16 & 0.5 & 0.333 \\
0.267 & 0.497 & 0.328 & 0.667 & 0.5
\end{array}\right) \\
\bar{F}^{2,0} & =\left(\begin{array}{ccccc}
0.5 & 0.125 & 0.562 & 0.062 & 0.062 \\
0.875 & 0.5 & 0.937 & 0.437 & 0.437 \\
0.438 & 0.063 & 0.5 & 0 & 0 \\
0.938 & 0.563 & 1 & 0.5 & 0.5 \\
0.938 & 0.563 & 1 & 0.5 & 0.5
\end{array}\right) \\
\bar{F}^{3,0} & =\left(\begin{array}{cccccc}
0.5 & 0.062 & 0.281 & 0 & 0.226 \\
0.938 & 0.5 & 0.718 & 0.437 & 0.663 \\
0.719 & 0.282 & 0.5 & 0.219 & 0.445 \\
1 & 0.563 & 0.781 & 0.5 & 0.726 \\
0.774 & 0.337 & 0.555 & 0.274 & 0.5
\end{array}\right) \\
\bar{F}^{4,0} & =\left(\begin{array}{cccccc}
0.5 & 0.5 & 0.5 & 0.75 & 0.25 \\
0.5 & 0.5 & 0.5 & 0.75 & 0.25 \\
0.5 & 0.5 & 0.5 & 0.75 & 0.25 \\
0.25 & 0.25 & 0.25 & 0.5 & 0 & \\
0.75 & 0.75 & 0.75 & 1 & 0.5
\end{array}\right) .
\end{aligned}
$$

Based on (3), the following consistency indices are obtained: $\mathrm{CI}\left(L^{1,0}\right)=0.817, \mathrm{CI}\left(L^{2,0}\right)=0.717, \mathrm{CI}\left(L^{3,0}\right)=$ 0.617 , and $\operatorname{CI}\left(L^{4,0}\right)=0.683$. These values are lower than the values obtained with PISs.

The corresponding consistent linguistic preference relations are

$\bar{L}^{1,0}=\left(\begin{array}{ccccc}\text { null } & \left(s_{4}, 0.477\right) & \left(s_{3}, 0.359\right) & \left(s_{5}, 0.401\right) & \left(s_{4}, 0.395\right) \\ \text { null } & \text { null } & \left(s_{2},-0.012\right) & \left(s_{4}, 0.018\right) & \left(s_{3}, 0.0005\right) \\ \text { null } & \text { null } & \text { null } & \left(s_{5}, 0.042\right) & \left(s_{4}, 0.03\right) \\ \text { null } & \text { null } & \text { null } & \text { null } & s_{2} \\ \text { null } & \text { null } & \text { null } & \text { null } & \text { null }\end{array}\right)$

$\bar{L}^{2,0}=\left(\begin{array}{ccccc}\text { null } & \left(s_{1},-0.251\right) & \left(s_{3}, 0.371\right) & \left(s_{0}, 0.371\right) & \left(s_{0}, 0.371\right) \\ \text { null } & \text { null } & \left(s_{6},-0.377\right) & \left(s_{3},-0.377\right) & \left(s_{3},-0.377\right) \\ \text { null } & \text { null } & \text { null } & s_{0} & s_{0} \\ \text { null } & \text { null } & \text { null } & \text { null } & s_{3} \\ \text { null } & \text { null } & \text { null } & \text { null } & \text { null }\end{array}\right)$

$\bar{L}^{3,0}=\left(\begin{array}{ccccc}\text { null } & \left(s_{0}, 0.371\right) & \left(s_{2},-0.311\right) & s_{0} & \left(s_{1}, 0.353\right) \\ \text { null } & \text { null } & \left(s_{4}, 0.305\right) & \left(s_{3},-0.377\right) & \left(s_{4},-0.024\right) \\ \text { null } & \text { null } & \text { null } & \left(s_{1}, 0.311\right) & \left(s_{3},-0.329\right) \\ \text { null } & \text { null } & \text { null } & \text { null } & \left(s_{4}, 0.353\right) \\ \text { null } & \text { null } & \text { null } & \text { null } & \text { null }\end{array}\right)$

$\bar{L}^{4,0}=\left(\begin{array}{ccccc}\text { null } & s_{3} & s_{3} & \left(s_{4}, 0.497\right) & \left(s_{1}, 0.497\right) \\ \text { null } & \text { null } & s_{3} & \left(s_{4}, 0.497\right) & \left(s_{1}, 0.497\right) \\ \text { null } & \text { null } & \text { null } & \left(s_{4}, 0.497\right) & \left(s_{1}, 0.497\right) \\ \text { null } & \text { null } & \text { null } & \text { null } & s_{0} \\ \text { null } & \text { null } & \text { null } & \text { null } & \text { null }\end{array}\right)$ 
The adjusted linguistic preference relations $L^{k, 1}(k=$ $1,2,3,4)$, which satisfy $l_{i j}^{k, 1} \in\left(l_{i j}^{k, 0}, \bar{l}_{i j}^{k, 0}\right]$, are

$$
\begin{aligned}
L^{1,1} & =\left(\begin{array}{ccccc}
\text { null } & s_{4} & s_{3} & s_{6} & s_{5} \\
\text { null } & \text { null } & s_{2} & s_{4} & s_{3} \\
\text { null } & \text { null } & \text { null } & s_{3} & s_{5} \\
\text { null } & \text { null } & \text { null } & \text { null } & s_{2} \\
\text { null } & \text { null } & \text { null } & \text { null } & \text { null }
\end{array}\right) \\
L^{2,1} & =\left(\begin{array}{ccccc}
\text { null } & s_{4} & s_{4} & s_{0} & s_{0} \\
\text { null } & \text { null } & s_{6} & s_{3} & s_{2} \\
\text { null } & \text { null } & \text { null } & s_{2} & s_{0} \\
\text { null } & \text { null } & \text { null } & \text { null } & s_{4} \\
\text { null } & \text { null } & \text { null } & \text { null } & \text { null }
\end{array}\right) \\
L^{3,1} & =\left(\begin{array}{llllc}
\text { null } & s_{0} & s_{3} & s_{0} & s_{3} \\
\text { null } & \text { null } & s_{4} & s_{4} & s_{2} \\
\text { null } & \text { null } & \text { null } & s_{2} & s_{2} \\
\text { null } & \text { null } & \text { null } & \text { null } & s_{5} \\
\text { null } & \text { null } & \text { null } & \text { null } & \text { null }
\end{array}\right) \\
L^{4,1} & =\left(\begin{array}{lcccc}
\text { null } & s_{2} & s_{4} & s_{5} & s_{2} \\
\text { null } & \text { null } & s_{3} & s_{5} & s_{1} \\
\text { null } & \text { null } & \text { null } & s_{4} & s_{2} \\
\text { null } & \text { null } & \text { null } & \text { null } & s_{3} \\
\text { null } & \text { null } & \text { null } & \text { null } & \text { null }
\end{array}\right) .
\end{aligned}
$$

The consistency indices are $\mathrm{CI}\left(L^{1,1}\right)=0.917, \mathrm{CI}\left(L^{2,1}\right)=$ $0.85, \mathrm{CI}\left(L^{3,1}\right)=0.85$, and $\mathrm{CI}\left(L^{4,1}\right)=0.883$. These values are lower than the values obtained based on PISs.

2) Second Iteration Without Considering PISs: The linguistic preference relations $L^{k, 1}(k=1,2,3,4)$ are transformed into their associated numerical preference relations, which are fed into Model (14), from which the following consistent numerical preference relations are obtained:

$$
\begin{aligned}
\bar{F}^{1,1} & =\left(\begin{array}{ccccc}
0.5 & 0.77 & 0.528 & 0.942 & 0.775 \\
0.23 & 0.5 & 0.258 & 0.672 & 0.505 \\
0.472 & 0.742 & 0.5 & 0.914 & 0.747 \\
0.058 & 0.328 & 0.086 & 0.5 & 0.333 \\
0.225 & 0.495 & 0.253 & 0.667 & 0.5
\end{array}\right) \\
\bar{F}^{2,1} & =\left(\begin{array}{ccccc}
0.5 & 0.131 & 0.631 & 0.131 & 0.131 \\
0.869 & 0.5 & 1 & 0.5 & 0.5 \\
0.369 & 0 & 0.5 & 0 & 0 \\
0.869 & 0.5 & 1 & 0.5 & 0.5 \\
0.869 & 0.5 & 1 & 0.5 & 0.5
\end{array}\right) \\
\bar{F}^{3,1} & =\left(\begin{array}{ccccc}
0.5 & 0.195 & 0.495 & 0.327 & 0.381 \\
0.805 & 0.5 & 0.8 & 0.631 & 0.686 \\
0.505 & 0.2 & 0.5 & 0.331 & 0.386 \\
0.673 & 0.369 & 0.669 & 0.5 & 0.555 \\
0.619 & 0.314 & 0.614 & 0.445 & 0.5
\end{array}\right) \\
\bar{F}^{4,1} & =\left(\begin{array}{ccccc}
0.5 & 0.503 & 0.652 & 0.827 & 0.448 \\
0.497 & 0.5 & 0.649 & 0.824 & 0.444 \\
0.348 & 0.351 & 0.5 & 0.675 & 0.296 \\
0.173 & 0.176 & 0.324 & 0.5 & 0.121 \\
0.552 & 0.556 & 0.704 & 0.879 & 0.5
\end{array}\right) .
\end{aligned}
$$

The corresponding consistent linguistic preference relations $\bar{L}^{k, 1}(k=1,2,3,4)$ are

$$
\begin{aligned}
\bar{L}^{1,1} & =\left(\begin{array}{llccc}
\text { null } & \left(s_{5},-0.377\right) & \left(s_{3}, 0.168\right) & \left(s_{6},-0.347\right) & \left(s_{5},-0.347\right) \\
\text { null } & \text { null } & \left(s_{2},-0.449\right) & \left(s_{4}, 0.03\right) & \left(s_{3}, 0.03\right) \\
\text { null } & \text { null } & \text { null } & \left(s_{5}, 0.485\right) & \left(s_{4}, 0.476\right) \\
\text { null } & \text { null } & \text { null } & \text { null } & s_{2} \\
\text { null } & \text { null } & \text { null } & \text { null } & \text { null }
\end{array}\right) \\
\bar{L}^{2,1} & =\left(\begin{array}{llcccc}
\text { null } & \left(s_{1},-0.215\right) & \left(s_{4},-0.215\right) & \left(s_{1},-0.215\right) & \left(s_{1},-0.215\right) \\
\text { null } & \text { null } & s_{6} & s_{3} & s_{3} \\
\text { null } & \text { null } & \text { null } & s_{0} & s_{0} \\
\text { null } & \text { null } & \text { null } & \text { null } & s_{3} \\
\text { null } & \text { null } & \text { null } & \text { null } & \text { null }
\end{array}\right) \\
\bar{L}^{3,1} & =\left(\begin{array}{llcccc}
\text { null } & \left(s_{1}, 0.168\right) & \left(s_{3},-0.03\right) & \left(s_{2},-0.036\right) & \left(s_{2}, 0.287\right) \\
\text { null } & \text { null } & \left(s_{5},-0.198\right) & \left(s_{4},-0.215\right) & \left(s_{4}, 0.114\right) \\
\text { null } & \text { null } & \text { null } & \left(s_{2},-0.012\right) & \left(s_{2}, 0.317\right) \\
\text { null } & \text { null } & \text { null } & \text { null } & \left(s_{3}, 0.329\right) \\
\text { null } & \text { null } & \text { null } & \text { null } & \text { null }
\end{array}\right) \\
\bar{L}^{4,1} & =\left(\begin{array}{llcccc}
\text { null } & \left(s_{3}, 0.018\right) & \left(s_{4},-0.09\right) & \left(s_{5},-0.036\right) & \left(s_{3},-0.311\right) \\
\text { null } & \text { null } & \left(s_{4},-0.108\right) & \left(s_{5},-0.054\right) & \left(s_{3},-0.335\right) \\
\text { null } & \text { null } & \text { null } & \left(s_{4}, 0.048\right) & \left(s_{2},-0.221\right) \\
\text { null } & \text { null } & \text { null } & \text { null } & \left(s_{1},-0.275\right) \\
\text { null } & \text { null } & \text { null } & \text { null } & \text { null }
\end{array}\right) .
\end{aligned}
$$

The adjusted linguistic preference relations $L^{k, 2}(k=$ $1,2,3,4)$, which satisfy $l_{i j}^{k, 2} \in\left(l_{i j}^{k, 1}, \bar{l}_{i j}^{k, 1}\right]$, are

$$
\begin{aligned}
L^{1,2} & =\left(\begin{array}{ccccc}
\text { null } & s_{4} & s_{3} & s_{6} & s_{5} \\
\text { null } & \text { null } & s_{2} & s_{4} & s_{3} \\
\text { null } & \text { null } & \text { null } & s_{5} & s_{5} \\
\text { null } & \text { null } & \text { null } & \text { null } & s_{2} \\
\text { null } & \text { null } & \text { null } & \text { null } & \text { null }
\end{array}\right) \\
L^{2,2} & =\left(\begin{array}{ccccc}
\text { null } & s_{3} & s_{4} & s_{0} & s_{0} \\
\text { null } & \text { null } & s_{6} & s_{3} & s_{3} \\
\text { null } & \text { null } & \text { null } & s_{1} & s_{0} \\
\text { null } & \text { null } & \text { null } & \text { null } & s_{4} \\
\text { null } & \text { null } & \text { null } & \text { null } & \text { null }
\end{array}\right) \\
L^{3,2} & =\left(\begin{array}{lllll}
\text { null } & s_{0} & s_{3} & s_{1} & s_{3} \\
\text { null } & \text { null } & s_{4} & s_{4} & s_{4} \\
\text { null } & \text { null } & \text { null } & s_{2} & s_{2} \\
\text { null } & \text { null } & \text { null } & \text { null } & s_{4} \\
\text { null } & \text { null } & \text { null } & \text { null } & \text { null }
\end{array}\right) \\
L^{4,2} & =\left(\begin{array}{lcccc}
\text { null } & s_{2} & s_{4} & s_{5} & s_{2} \\
\text { null } & \text { null } & s_{3} & s_{5} & s_{2} \\
\text { null } & \text { null } & \text { null } & s_{4} & s_{2} \\
\text { null } & \text { null } & \text { null } & \text { null } & s_{1} \\
\text { null } & \text { null } & \text { null } & \text { null } & \text { null }
\end{array}\right) .
\end{aligned}
$$

The consistency indices obtained are $\mathrm{CI}\left(L^{1,2}\right)=0.95$, $\mathrm{CI}\left(L^{2,2}\right)=0.9, \mathrm{CI}\left(L^{3,2}\right)=0.917$, and $\mathrm{CI}\left(L^{4,2}\right)=0.917$. These values are again lower than the values obtained with PISs.

Both Algorithms 1 and 2 improve the consistency of linguistic preference relations, being the improvement higher with PISs (Algorithm 1) than without PISs (Algorithm 2). In the next section, the difference between the two algorithms will be further analyzed with a simulation analysis. 

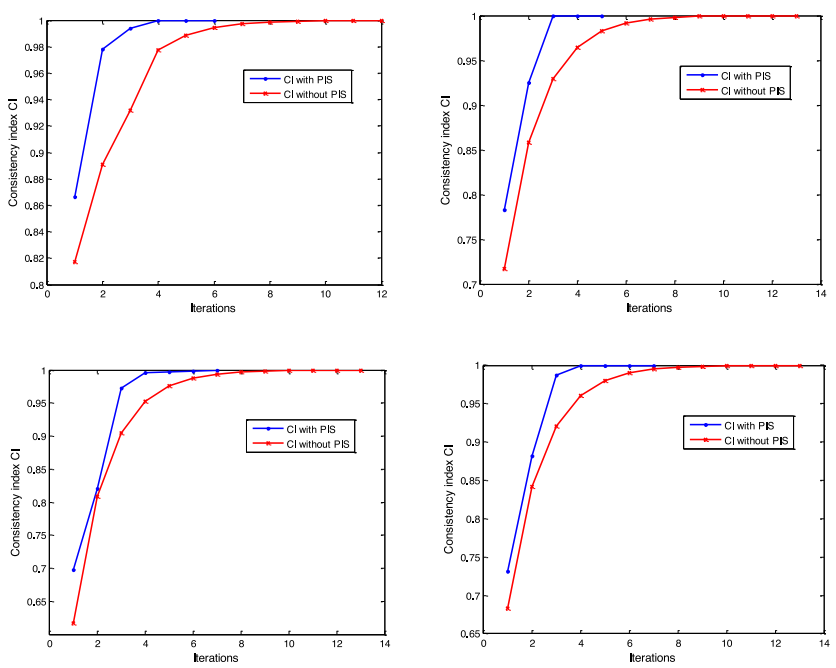

Fig. 3. Consistency improvement process for $L^{k}(k=1,2,3,4)$ for Algorithms 1 and $2(\gamma=0.5)$.
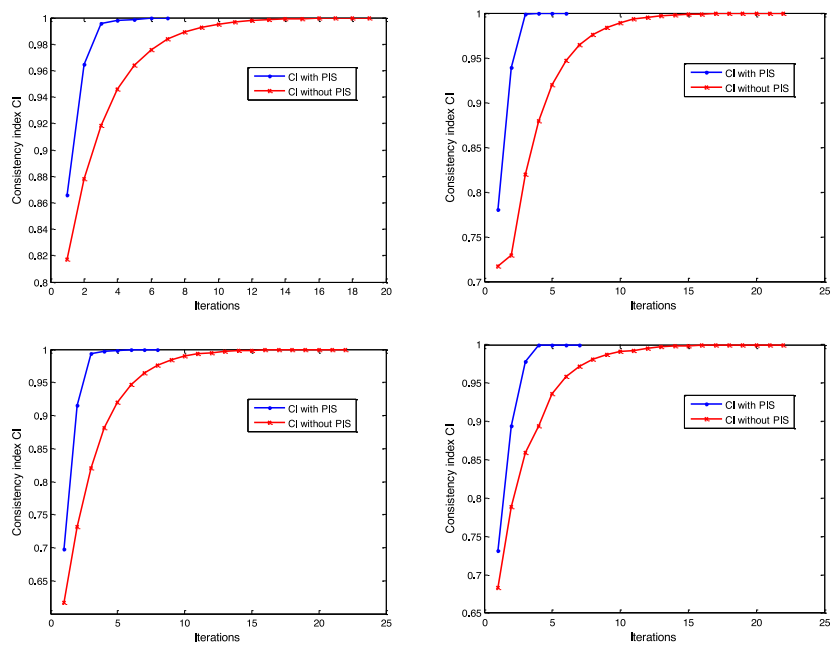

Fig. 4. Process to improve consistency of $L^{k}(k=1,2,3,4)$ based on Algorithms 1 and $2(\gamma=(1 / 3))$.

\section{B. Simulation Analysis}

A simulation analysis to explore the speed of convergence to consistency of the linguistic preference relations by both Algorithms is given below. To automatically change the preferences of decision makers, (13) and (15) in Algorithms 1 and 2 are replaced with (16) and (17), respectively

$$
\begin{aligned}
& l_{i j}^{k, t+1}=\mathrm{NS}^{-1}\left(\gamma \times \mathrm{NS}\left(l_{i j}^{k, t}\right)+(1-\gamma) \times \mathrm{NS}\left(\bar{l}_{i j}^{k, t}\right)\right), \quad \gamma \in[0,1) \\
& l_{i j}^{k, t+1}=\Delta\left(\gamma \times \Delta^{-1}\left(l_{i j}^{k, t}\right)+(1-\gamma) \times \Delta^{-1}\left(\bar{l}_{i j}^{k, t}\right)\right), \quad \gamma \in[0,1) .
\end{aligned}
$$

The same linguistic preference relations $L^{k}(k=1,2,3,4)$ provided in Section IV are used with values $\gamma=0.5 ; \gamma=$ $(1 / 3)$; and $\gamma=(2 / 3)$. The consistency variation of $L^{k}(k=$ $1,2,3,4)$ using Algorithm 1 and Algorithm 2 is depicted in Figs. 3-5, respectively.

\section{Lessons Learned}

The following observations are drawn.
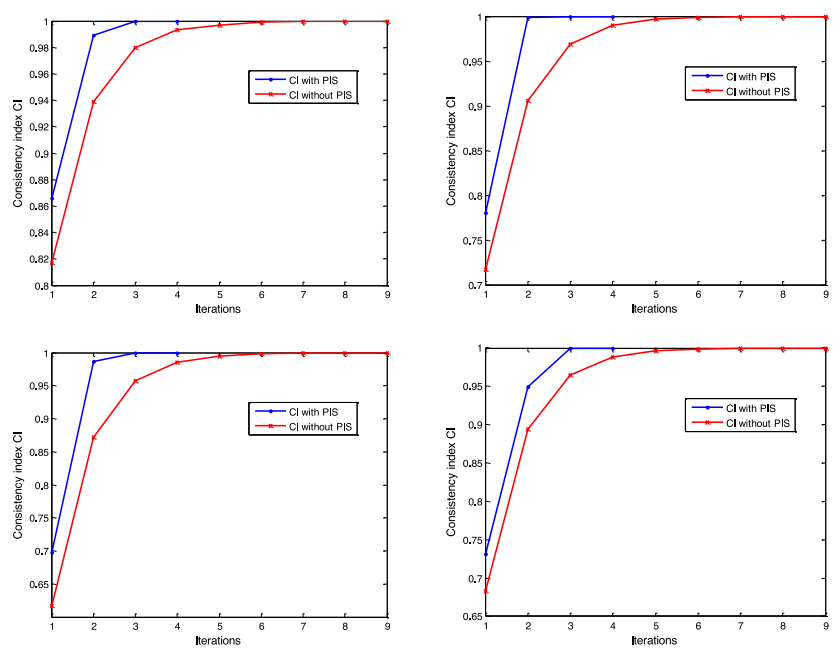

Fig. 5. Process to improve consistency of $L^{k}(k=1,2,3,4)$ based on Algorithms 1 and $2(\gamma=(2 / 3))$.

1) The consistency levels of linguistic preference relations improve with both Algorithms. The improvement process is increasing, and because of their boundedness property, it is convergent.

2) Algorithm 1 improves consistency more rapidly than Algorithm 2. For $\gamma=0.5$, the consistency index reaches 1 in less than six iterations of Algorithm 1, while it takes 12 iterations of Algorithm 2. For $\gamma=(1 / 3)$ and $\gamma=(2 / 3)$, the consistency index reaches 1 in about 5 and 3 iterations of Algorithm 1, respectively, while it requires about 20 and 9 iterations of Algorithm 2, respectively.

3) The number of iterations required for the consistency index to reach 1 decrease when the value of $\gamma$ increases.

The above observations show that the implementation of PISs can improve consistency in GDM effectively. Particularly, from the comparisons with Algorithm 2, the PIS-based approach shows that personalized numerical meanings of words can help decision makers achieving personalized adjusted linguistic preference relations with acceptable consistency more rapidly.

\section{CONCLUSION}

The use of PISs in linguistic GDM provides a new avenue for studying consistency issues. In this article, a novel PIS-based consistency index for linguistic preference relations is being introduced. By integrating a consistency-driven optimization model, an iterative algorithm with PISs has been developed to improve the consistency of linguistic preference relations. Finally, we provide numerical analysis to illustrate the application of the proposed model and report on a detailed simulated analysis of the differences between the consistency improving process of the proposed PIS-based approach and the corresponding 2-tuple linguistic model approach that does not implement PISs. The implementation of PISs leads to higher increasers of consistency and a more rapid convergence to the established consistency level than that when PISs are not considered. Therefore, the PIS-based method provides a useful 
tool to measure the consistency with PISs and to improve the consistency degree of linguistic preference relations.

Although the PIS-based method is performing well to manage the consistency measurement and improvement with linguistic preference relations, in GDM, more complex linguistic environments than the research in this article exist. These are based on the use of hesitant linguistic term sets [29], [39]; linguistic distribution [41]; multigranular linguistic term set [31]; and flexible linguistic expressions [37]. In the future, we will further study PIS-based approaches to consistency issues in such complex linguistic environments.

\section{REFERENCES}

[1] S. Alonso, F. Chiclana, F. Herrera, E. Herrera-Viedma, J. Alcalá-Fdez, and C. Porcel, "A consistency-based procedure to estimate missing pairwise preference values," Int. J. Intell. Syst., vol. 23, no. 2, pp. $155-175,2008$.

[2] B. Chandran, B. Golden, and E. Wasil, "Linear programming models forestimating weights in the analytic hierarchy process," Comput. Oper. Res., vol. 32, no. 9, pp. 2235-2254, 2005.

[3] G. Crawford and C. Williams, "A note on the analysis of subjective judgment matrices," J. Math. Psychol., vol. 29, no. 4, pp. 387-405, 1985.

[4] Y. C. Dong, Y. Xu, and S. Yu, "Computing the numerical scale of the linguistic term set for the 2-tuple fuzzy linguistic representation model," IEEE Trans. Fuzzy Syst., vol. 17, no. 6, pp. 1366-1378, Dec. 2009.

[5] Y. C. Dong, C.-C. Li, and F. Herrera, "Connecting the linguistic hierarchy and the numerical scale for the 2-tuple linguistic model and its use to deal with hesitant unbalanced linguistic information," Inf. Sci., vols. 367-368, pp. 259-278, Nov. 2016.

[6] Y. C. Dong, Y. Xu, and H. Li, "On consistency measures of linguistic preference relations," Eur. J. Oper. Res., vol. 189, no. 2, pp. 430-444, 2008.

[7] Y. C. Dong and E. Herrera-Viedma, "Consistency-driven automatic methodology to set interval numerical scales of 2-tuple linguistic term sets and its use in the linguistic GDM with preference relations," IEEE Trans. Cybern., vol. 45, no. 5, pp. 780-792, Apr. 2015.

[8] Z. Gong, W. Guo, E. Herrera-Viedma, Z. Gong, and G. Wei, "Consistency and consensus modeling of linear uncertain preference relations," Eur. J. Oper. Res., vol. 283, no. 1, pp. 290-307, 2020.

[9] F. Herrera and E. Herrera-Viedma, "Choice functions and mechanisms for linguistic preference relations," Eur. J. Oper. Res., vol. 120, no. 1, pp. 144-161, 2000.

[10] F. Herrera and L. Martínez, "A 2-tuple fuzzy linguistic representation model for computing with words," IEEE Trans. Fuzzy Syst., vol. 8, no. 6, pp. 746-752, Dec. 2000.

[11] F. Herrera, S. Alonso, F. Chiclana, and E. Herrera-Viedma, "Computing with words in decision making: Foundations, trends and prospects," Fuzzy Optim. Decis. Mag., vol. 8, pp. 337-364, Sep. 2009.

[12] F. Herrera, E. Herrera-Viedma, and J. L. Verdegay, "Preference degrees over linguistic preference relations in decision making," Badania Operacyjne I Decyzje Operational Res. Decis., vol. 3, pp. 37-48, 1995.

[13] F. Herrera, E. Herrera-Viedma, and J. L. Verdegay, "A sequential selection process in group decision making with a linguistic assessment approach," Inf. Sci., vol. 85, no. 4, pp. 223-239, 1995.

[14] F. Herrera and L. Martínez, "A model based on linguistic 2-tuples for dealing with multigranular hierarchical linguistic context in multi-expert decision making," IEEE Trans. Syst. Man, Cybern. B, Cybern., vol. 31, no. 2, pp. 227-234, Apr. 2001.

[15] F. Herrera, E. Herrera-Viedma, and L. Martínez, "A fuzzy linguistic methodology to deal with unbalanced linguistic term sets," IEEE Trans. Fuzzy Syst., vol. 16, no. 2, pp. 354-370, Apr. 2008.

[16] E. Herrera-Viedma, F. Chiclana, F. Herrera, and S. Alonso, "Group decision-making model with incomplete fuzzy preference relations based on additive consistency," IEEE Trans. Syst. Man, Cybern. B, Cybern., vol. 37, no. 1, pp. 176-189, Feb. 2007.

[17] F. Jin, Z. Ni, H. Chen, and Y. Li, "Approaches to decision making with linguistic preference relations based on additive consistency," Appl. Soft Comput., vol. 49, pp. 71-80, Dec. 2016.

[18] G. Kou, D. Ergu, and J. Shang, "Enhancing data consistency in decision matrix: Adapting Hadamard model to mitigate judgment contradiction," Eur. J. Oper. Res., vol. 236, no. 1, pp. 261-271, 2014.
[19] C. C. Li, Y. C. Dong, F. Herrera, E. Herrera-Viedma, and L. Martínez, "Personalized individual semantics in computing with words for supporting linguistic group decision making. An application on consensus reaching," Inf. Fusion, vol. 33, pp. 29-40, Jan. 2017.

[20] C.-C. Li, R. M. Rodríguez, L. Martínez, Y. C. Dong, and F. Herrera, "Personalized individual semantics based on consistency in hesitant linguistic group decision making with comparative linguistic expressions," Knowl. Based Syst., vol. 145, pp. 156-165, Apr. 2018.

[21] C.-C. Li, Y. C. Dong, Y. X. Xu, F. Chiclana, E. Herrera-Viedma, and F. Herrera, "An overview on managing additive consistency of reciprocal preference relations for consistency-driven decision making and fusion: Taxonomy and future directions," Inf. Fusion, vol. 52, pp. 143-156, Dec. 2019.

[22] C.-C. Li, Y. C. Dong, and F. Herrera, "A consensus model for largescale linguistic group decision making with a feedback recommendation based on clustered personalized individual semantics and opposing consensus groups," IEEE Trans. Fuzzy Syst., vol. 27, no. 2, pp. 221-233, Feb. 2019.

[23] H. Liang, C.-C. Li, Y. C. Dong, and F. Herrera, "Linguistic opinions dynamics based on personalized individual semantics," IEEE Trans. Fuzzy Syst., early access, Jun. 3, 2020, doi: 10.1109/TFUZZ.2020.2999742.

[24] F. Liu and W.-G. Zhang, "TOPSIS-based consensus model for group decision-making with incomplete interval fuzzy preference relations," IEEE Trans. Cybern., vol. 44, no. 8, pp. 1283-1294, Aug. 2014.

[25] L. Fang, P. Witold, and L. Xin-Wang, "Flexibility degree of fuzzy numbers and its implication to a group-decision-making model," IEEE Trans. Cybern., vol. 49, no. 12, pp. 4054-4065, Dec. 2019.

[26] J. M. Mendel and D. Wu, Perceptual Computing: Aiding People in Making Subjective Judgments. New York, NY, USA: Wiley, 2010.

[27] J. M. Mendel et al., "What computing with words means to me: Discussion forum," IEEE Comput. Intell. Mag., vol. 5, no. 1, pp. 20-26, Feb. 2010.

[28] J. A. Morente-Molinera, I. J. Pérez, M. R. Urea, and E. Herrera-Viedma, "On multi-granular fuzzy linguistic modelling in group decision making problems: A systematic review and future trends," Knowl. Based Syst., vol. 74, pp. 49-60, Jan. 2015.

[29] R. M. Rodríguez, L. Martínez, and F. Herrera, "Hesitant fuzzy linguistic term sets for decision making," IEEE Trans. Fuzzy Syst., vol. 20, no. 1, pp. 109-119, Feb. 2012.

[30] T. L. Saaty, The Analytic Hierarchy Process. New York, NY, USA: McGraw Hill, 1980, pp. 9-26.

[31] Y. M. Song and G. X. Li, "A large-scale group decision-making with incomplete multi-granular probabilistic linguistic term sets and its application in sustainable supplier selection," J. Oper. Res. Soc., vol. 70, no. 5, pp. 827-841, 2019.

[32] T. Tanino, "Fuzzy preference orderings in group decision making," Fuzzy Sets Syst., vol. 12, no. 2, pp. 117-131, 1984.

[33] T. Tanino, "Fuzzy preference relations in group decision making," in Non-Conventional Preference Relations in Decision Making, J. Kacprzyk and M. Roubens, Eds. Heidelberg, Germany: Springer-Verlag, 1988, pp. 54-71.

[34] X. Tang, Q. Zhang, Z. Peng, S. Yang, and W. Pedrycz, "Derivation of personalized numerical scales from distribution linguistic preference relations: An expected consistency-based goal programming approach," Neural Comput. Appl., vol. 31, no. 12, pp. 8769-8786, 2019.

[35] X. Tang, Q. Zhang, Z. Peng, W. Pedrycz, and S. Yang, "Distribution linguistic preference relations with incomplete symbolic proportions for group decision making," Appl. Soft Comput., vol. 88, Mar. 2020, Art. no. 106005.

[36] J. H. Wang and J. Hao, "A new version of 2-tuple fuzzy linguistic representation model for computing with words," IEEE Trans. Fuzzy Syst., vol. 14, no. 3, pp. 435-445, Jun. 2006.

[37] Y. Z. Wu, Y. C. Dong, J. Qin, and W. Pedrycz, "Flexible linguistic expressions and consensus reaching with accurate constraints in group decision-making," IEEE Trans. Cybern., vol. 50, no. 6, pp. 2488-2501, Jun. 2020

[38] Z. Wu, S. Huang, and J. Xu, "Multi-stage optimization models for individual consistency and group consensus with preference relations," Eur. J. Oper. Res., vol. 275, no. 1, pp. 182-194, 2019.

[39] Z. B. Wu and J. P. Xu, "Possibility distribution-based approach for MAGDM with hesitant fuzzy linguistic information," IEEE Trans. Cybern., vol. 46, no. 3, pp. 694-705, Mar. 2016.

[40] P. Wu, J. P. Liu, L. G. Zhou, and H. Y. Chen, "Algorithm for improving additive consistency of linguistic preference relations with an integer optimization model," Appl. Soft Comput., vol. 86, Jan. 2020, Art. no. 105955. 
[41] G. Q. Zhang, Y. C. Dong, and Y. F. Xu, "Consistency and consensus measures for linguistic preference relations based on distribution assessments," Inf. Fusion, vol. 17, pp. 46-55, May 2014.

[42] Z. Zhang, X. Y. Kou, W. Y. Yu, and Y. Gao, "Consistency improvement for fuzzy preference relations with self-confidence: An application in two-sided matching decision making," J. Oper. Res. Soc., to be published, doi: 10.1080/01605682.2020.1748529.

[43] H. Zhang, C.-C. Li, Y. Liu, and Y. C. Dong, "Modelling personalized individual semantics and consensus in comparative linguistic expression preference relations with self-confidence: An optimizationbased approach," IEEE Trans. Fuzzy Syst., vol. 29, no. 3, pp. 627-640, Mar. 2021, doi: 10.1109/TFUZZ.2019.2957259.

[44] H. Zhang, Y. C. Dong, J. Xiao, F. Chiclana, and E. Herrera-Viedma, "Personalized individual semantics-based approach for linguistic failure modes and effects analysis with incomplete preference information," IISE Trans., vol. 52, no. 11, pp. 1275-1296, 2020, doi: $10.1080 / 24725854.2020 .1731774$.

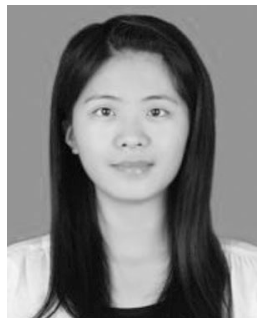

Cong-Cong $\mathbf{L i}$ received the Ph.D. degree in management from Sichuan University, Chengdu, China, in 2018, and the Ph.D. degree in computer science and artificial intelligence from the University of Granada, Granada, Spain, in 2018.

She is currently a Lecturer with the School of Economics and Management, Southwest Jiaotong University, Chengdu. Her research interests include decision making, computing with words, and opinion dynamics. Her research results have been published in some refereed journals, including IEEE TRANSACTIONS ON FUZZY SYstems, Information Fusion, Information Sciences, Knowledge-Based Systems, and Group Decision and Negotiation.

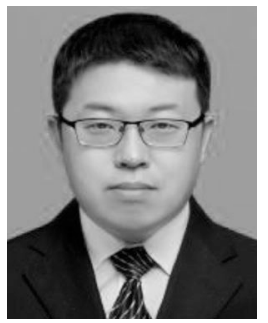

Haiming Liang received the Ph.D. degree in management from Northeastern University, Shenyang, China, in 2014.

$\mathrm{He}$ is an Associate Research Fellow with the Business School, Sichuan University, Chengdu, China. He published several international journal papers in European Journal of Operational Research, Omega, Computers \& Industrial Engineering, Information Fusion, Journal of Artificial Societies and Social Simulation, and Journal of the Operational Research Society. His research interests include multiple attribute decision making and opinion dynamics.

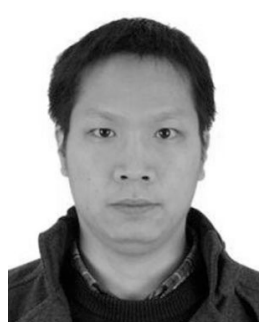

Yucheng Dong (Senior Member, IEEE) received the B.S. and M.S. degrees in mathematics from Chongqing University, Chongqing, China, in 2002 and 2004, respectively, and the Ph.D. degree in management from Xi'an Jiaotong University, Xi'an, China, in 2008 .

$\mathrm{He}$ is currently a Professor with the Business School, Sichuan University, Chengdu, China. He has authored or coauthored more than 100 international journal papers in some refereed journals. His current research interests include decision analysis, human dynamics, big data analytics, social network, and risk analysis.

Prof. Dong is an Area Editor/Associate Editor of Computers and Industrial Engineering, Group Decision and Negotiation, the IEEE TRANSACTIONS ON Systems, MAN, AND CYBERNETICS: Systems, and Information Fusion. He has been identified by Clarivate as a Highly Cited Researcher in the field of computer science.

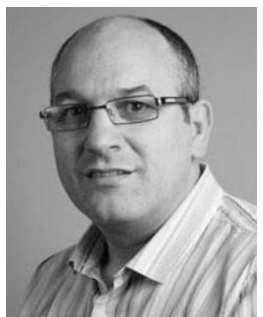

Francisco Chiclana received the B.Sc. and Ph.D. degrees in mathematics from the University of Granada, Granada, Spain, in 1989 and 2000, respectively.

He is currently a Professor of Computational Intelligence and Decision Making with the School of Computer Science and Informatics, Faculty of Technology, De Montfort University, Leicester, U.K., and was an Honorary Professor with the Department of Mathematics, University of Leicester, Leicester, U.K., from 2015 to 2018 . He has published extensively in leading international journals with more than 70 journal articles indexed in Web of Science. His current research interests include decision making, computational intelligence, information fusion, consensus, rule induction, and sentiment analysis.

Prof. Chiclana is an Associate Editor of the IEEE TRANSACTIONS ON Systems, MAN, AND CYBERNETICS: Systems, Information Sciences, Soft Computing, the International Journal of Fuzzy Systems, and the Journal of Intelligent and Fuzzy Systems. He has also been consistently classified as one of the Most Cited Scientists in its field by ESI. He also serves as a member of the Editorial Board of Applied Soft Computing, Knowledge-Based Systems, and Multiple-Valued Logic and Soft Computing.

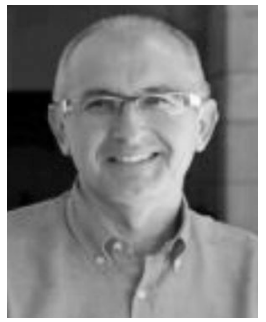

Enrique Herrera-Viedma (Fellow, IEEE) received the M.Sc. and Ph.D. degrees in computer science from the University of Granada, Granada, Spain, in 1993 and 1996, respectively.

$\mathrm{He}$ is a Professor of Computer Science and the Vice-President for Research and Knowledge Transfer with the University of Granada. His H-index is 82 with more than 24000 citations received in Web of Science and 100 in Google Scholar with more than 40000 citations received. His current research interests include group decision making, consensus models, linguistic modeling, aggregation of information, information retrieval, bibliometric, digital libraries, Web quality evaluation, recommender systems, and social media.

Prof. Herrera-Viedma is the Vice-President for Cybernetics in IEEE SMC and an Associate Editor in several journals, such as IEEE TRANSACTIONS on FuZZy Systems, IEEE TRANSACTIONS ON SySTEMS, MAN, AND Cybernetics: Systems, IEEE TRANSACTIONS ON INTELligent Transportation System, Information Sciences, Applied Soft Computing, Soft Computing, Fuzzy Optimization and Decision Making, and KnowledgeBased Systems. He has been identified as one of the World's Most Influential Researchers by the Shanghai Center and Thomson Reuters/Clarivate Analytics in both computer science and engineering from 2014 to 2020. 